\title{
THE SUBLUMINOUS AND PECULIAR TYPE Ia SUPERNOVA PTF 09dav
}

\author{
M. Sullivan ${ }^{1}$, M. M. Kasliwal ${ }^{2}$, P. E. Nugent ${ }^{3}$, D. A. Howell ${ }^{4,5}$, R. C. Thomas ${ }^{3}$, E. O. OfeK ${ }^{1}$ I. Arcavi $^{6}$, S. Blake $^{1}$, \\ J. Cooke ${ }^{2}$, A. Gal-Yam ${ }^{6}$, I. M. Hook ${ }^{7}$, P. Mazzali ${ }^{8,9,10}$, P. Podsiadlowski ${ }^{1}$, R. QuimbY ${ }^{2}$ L. Bildsten $^{5,11}$, J. S. Bloom ${ }^{12}$, \\ S. B. Cenko ${ }^{12}$, S. R. Kulkarni ${ }^{2}$, N. Law ${ }^{13}$, AND D. PoznAnski ${ }^{3,12}$ \\ ${ }^{1}$ Department of Physics (Astrophysics), University of Oxford, Keble Road, Oxford OX1 3RH, UK; sullivan@ astro.ox.ac.uk \\ ${ }^{2}$ Cahill Center for Astrophysics, California Institute of Technology, Pasadena, CA 91125, USA \\ ${ }^{3}$ Computational Cosmology Center, Lawrence Berkeley National Laboratory, 1 Cyclotron Road, Berkeley, CA 94720, USA \\ ${ }^{4}$ Las Cumbres Observatory Global Telescope Network, 6740 Cortona Drive, Suite 102, Goleta, CA 93117, USA \\ ${ }^{5}$ Department of Physics, University of California Santa Barbara, Santa Barbara, CA 93106, USA \\ ${ }^{6}$ Department of Particle Physics and Astrophysics, Faculty of Physics, The Weizmann Institute of Science, Rehovot 76100, Israel \\ ${ }^{7}$ INAF-Osservatorio di Roma, via Frascati 33, I-00040 Monteporzio Catone (Roma), Italy \\ ${ }^{8}$ Max-Planck-Institut für Astrophysik, Karl-Schwarzschild-Str. 1, 85741 Garching, Germany \\ ${ }^{9}$ Scuola Normale Superiore, Piazza dei Cavalieri 7, 56126 Pisa, Italy \\ ${ }^{10}$ National Institute for Astrophysics-OAPd, Vicolo dell'Osservatorio 5, 35122 Padova, Italy \\ ${ }^{11}$ Kavli Institute for Theoretical Physics, University of California Santa Barbara, Santa Barbara, CA 93106, USA \\ 12 Department of Astronomy, University of California, Berkeley, CA 94720-3411, USA \\ ${ }^{13}$ Dunlap Institute for Astronomy and Astrophysics, University of Toronto, 50 St. George Street, Toronto M5S 3H4, Ontario, Canada \\ Received 2010 December 12; accepted 2011 March 9; published 2011 April 26
}

\begin{abstract}
PTF 09dav is a peculiar subluminous Type Ia supernova (SN) discovered by the Palomar Transient Factory (PTF). Spectroscopically, it appears superficially similar to the class of subluminous SN1991bg-like SNe, but it has several unusual features which make it stand out from this population. Its peak luminosity is fainter than any previously discovered SN1991bg-like SN Ia $\left(M_{B} \sim-15.5\right)$, but without the unusually red optical colors expected if the faint luminosity were due to extinction. The photospheric optical spectra have very unusual strong lines of Sc II and $\mathrm{Mg} \mathrm{I}$, with possible Sr II, together with stronger than average Ti II and low velocities of $\sim 6000 \mathrm{~km} \mathrm{~s}^{-1}$. The host galaxy of PTF09dav is ambiguous. The SN lies either on the extreme outskirts $(\sim 41 \mathrm{kpc})$ of a spiral galaxy or in an very faint $\left(M_{R} \geqslant-12.8\right)$ dwarf galaxy, unlike other $1991 \mathrm{bg}$-like SNe which are invariably associated with massive, old stellar populations. PTF 09dav is also an outlier on the light-curve-width-luminosity and color-luminosity relations derived for other subluminous SNe Ia. The inferred ${ }^{56} \mathrm{Ni}$ mass is small $\left(0.019 \pm 0.003 M_{\odot}\right)$, as is the estimated ejecta mass of $0.36 M_{\odot}$. Taken together, these properties make PTF 09dav a remarkable event. We discuss various physical models that could explain PTF 09dav. Helium shell detonation or deflagration on the surface of a CO white dwarf can explain some of the features of PTF 09dav, including the presence of Sc and the low photospheric velocities, but the observed $\mathrm{Si}$ and $\mathrm{Mg}$ are not predicted to be very abundant in these models. We conclude that no single model is currently capable of explaining all of the observed signatures of PTF 09dav.
\end{abstract}

Key words: supernovae: general - supernovae: individual (PTF09dav)

Online-only material: color figures

\section{INTRODUCTION}

Type Ia supernovae ( $\mathrm{SNe}$ Ia), thermonuclear explosions of accreting carbon-oxygen white dwarf stars, form a fairly uniform and homogeneous class of events. This has inspired their application as cosmological standardizable candles, tracing the cosmic expansion history to high redshift. However, the class of "subluminous" SN Ia events show quite different photometric and spectroscopic properties than those mainstream events used for cosmological studies, with potentially different progenitor models. Though the discovery of these rare, faint SNe Ia has historically been challenging, the archetypal subluminous SN1991bg (Filippenko et al. 1992; Leibundgut et al. 1993) has been complemented with the discovery of many similar events (e.g., see compilations of Branch et al. 1993; Garnavich et al. 2004; Taubenberger et al. 2008; Gonzalez-Gaitan et al. 2011). Modern SN searches are revealing ever more extreme examples of faint $\mathrm{SNe}$ of all types, the nature of which, in some cases, remains uncertain (e.g., Li et al. 2003; Kasliwal et al. 2008; Valenti et al. 2009; Foley et al. 2009; Perets et al. 2010; Foley et al. 2010; McClelland et al. 2010; Kasliwal et al. 2010).

The key characteristics of the 1991bg-like subluminous SN Ia population are a faint peak absolute magnitude $(\sim 2$ magnitudes fainter than a normal SN Ia) with a red optical color, a light curve that rises and falls $\sim 40 \%$ faster than a normal SN Ia, a strong trend of being located in old, E/S0 galaxies with large stellar masses (Howell 2001; Neill et al. 2009), and "cool" optical maximum light spectra dominated by strong Ti II absorption (Filippenko et al. 1992; Mazzali et al. 1997; Branch et al. 2006) with somewhat lower expansion velocities than in normal SNe Ia (Benetti et al. 2005). The class of SNe Ia similar to SN2002cx (Li et al. 2003) appear to form a further, separate sub-class of faint events, with a host galaxy distribution favoring later morphological types compared to that of SN1991bg-like events (Foley et al. 2009; Valenti et al. 2009). Some events in the subclass, such as SN2008ha, are fainter still than 1991bg-like SNe (Foley et al. 2009; McClelland et al. 2010), with even lower ejecta velocities (and kinetic energies). Recent observations also demonstrate the existence of further faint or fast types of SN events, where the formal SN type classification may provide misleading information about the physical nature of the explosion (Poznanski et al. 2010; Perets et al. 2010, 2011).

Various physical models and scenarios have been proposed to explain subluminous SN1991bg-like events. Whether they form a distinct physical group from normal $\mathrm{SNe}$ Ia, with different progenitors and explosion models, or whether they lie 
at the extreme end of a continuous distribution, but with lower

${ }^{56} \mathrm{Ni}$ masses and hence temperatures, is unclear. No conclusive evidence for any one progenitor model has so far been found. The nature of the progenitors of SN2002cx-like events is even less clear, with possible models ranging from the direct collapse of a massive star to a black hole (a "fallback" SN; Valenti et al. 2009; Moriya et al. 2010) to the pure deflagration of a white dwarf (e.g., Branch et al. 2004; Phillips et al. 2007).

In this paper we describe the supernova PTF 09dav. This SN was discovered as part of the Palomar Transient Factory $\left(\mathrm{PTF}^{14}\right.$; Rau et al. 2009; Law et al. 2009), a five-year project surveying the optically transient sky. PTF is designed to sample a large fraction of the optical transient population, including both new types of events, as well as statistically complete samples of known transient types. In particular, the high-cadence, survey depth, and wide-area make PTF ideal for the study of fast, subluminous SN events (e.g., Kasliwal et al. 2010). PTF 09dav is one such event-a very subluminous SN Ia with peculiar (and so far unique) spectral properties. In Section 2, we introduce PTF 09dav together with its basic photometric, spectral, and host galaxy data. We analyze the light curve in Section 3, deriving estimates of its peak luminosity and related parameters. Section 4 details our spectral analysis, including the identification of the principal elements. We discuss various physical interpretations of our data in Section 5. In a companion paper, we discuss the late-time light curve and nebular spectroscopic data for this event (M. M. Kasliwal et al. 2011, in preparation). Where relevant we assume $H_{0}=70 \mathrm{~km} \mathrm{~s}^{-1} \mathrm{Mpc}^{-1}$.

\section{OBSERVATIONS}

PTF 09dav was discovered as a transient event at $R \sim 19.5$ by the PTF on 2009 August 11.3 (all dates in this paper are UTC), located at $\alpha=22^{\mathrm{h}} 46^{\mathrm{m}} 55^{\mathrm{s}} .15, \delta=+21^{\circ} 37^{\prime} 34^{\prime \prime} .1$ (J2000; Figure 1). The event was isolated with no apparent host galaxy in the PTF reference images. A spectrum was taken with the William Herschel Telescope (WHT) using the Intermediate dispersion Spectrograph and Imaging System on 2009 August 14.0 as part of the PTF SN Ia key project. The R316R (red arm) and R300B (blue arm) gratings were used, together with the 5300 dichroic, giving a wavelength coverage of 3400-8100 ̊. Spectral comparisons were performed at the telescope using the SUPERFIT spectral matching code (e.g., Howell et al. 2005) and the spectrum was initially recognized as being superficially similar to the subluminous SN Ia SN1991bg (Filippenko et al. 1992), with deep Ti II, Si II, and O I absorption, located at a redshift of $z \sim 0.04$ (Figure 2). Given the faint discovery magnitude and relatively low redshift, PTF 09dav was recognized as a potentially rare and interesting subluminous SN event and was triggered for further study, which we summarize in the remainder of this section.

\subsection{Spectra}

Following the initial WHT classification spectrum, two further spectra were taken during the photospheric phase of the SN. The first was taken with the Palomar Hale 200 inch telescope (P200) plus Double Beam Spectrograph (DBSP; Oke \& Gunn 1982) on 2009 August 20.5. The 6001/4000 A (blue) and 1581/ $7500 \AA$ (red) gratings, and D55 dichroic, were used, giving a wavelength coverage of $\sim 3500-9500 \AA$. The second spectrum was taken using the Keck I telescope and the Low-Resolution

\footnotetext{
14 http://www.astro.caltech.edu/ptf/
}

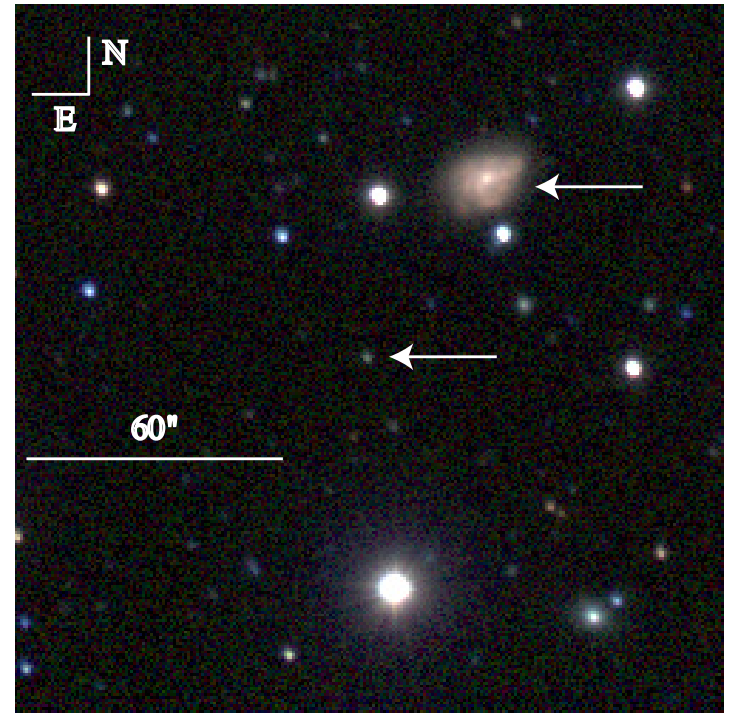

Figure 1. False color image constructed from P60 $g, r$, and $i$ data showing the location of PTF 09dav in relation to the nearby "host galaxy." The upper arrow indicates the putative host and the lower arrow the SN. The separation is 56.8 , which at $z_{\mathrm{cmb}}=0.0359$ corresponds to $40.6 \mathrm{kpc}$. The host galaxy has an apparently disturbed morphology, and a spectrum shows it to be dominated by nebular emission lines indicative of ongoing star formation. No host is detected at the position of PTF 09dav to $m_{\mathrm{RG} 610}=23.2\left(M_{\mathrm{RG} 610}=-12.8\right)$.

Imaging Spectrometer (Oke et al. 1995) on 2009 August 25.5-a wavelength coverage of 3400-10000 $\AA$ was achieved using the 4001/3400 ̊ grism (blue), 4001/8500 A grating (red), and the 560 dichroic. A final spectrum taken during the nebular phase of the SN was taken on 2009 November 11.4 and will be presented in a companion paper (M. M. Kasliwal et al. 2010, in preparation).

The three photospheric spectra were reduced using the same custom-written pipeline based on standard procedures in IRAF and IDL broadly following the reduction procedures outlined in Ellis et al. (2008), including flux calibration and telluric feature removal. All spectra were observed at the parallactic angle in photometric conditions. "Error" spectra are derived from a knowledge of the CCD properties and Poisson statistics and are tracked throughout the reduction procedure. The spectra are also rebinned (in a weighted way) to a common dispersion and joined across the dichroic. The three spectra are shown in Figures 2 and 3.

\subsection{Host Galaxy}

The host galaxy of PTF 09dav is somewhat ambiguous (Figure 1). PTF 09dav lies 56".8 (projected) SE of a potential host galaxy $\left(r \simeq 17.6 ; \alpha=22^{\mathrm{h}} 46^{\mathrm{m}} 52^{\mathrm{s}} .9, \delta=+21^{\circ} 38^{\prime} 21^{\prime \prime} .6\right.$, J2000). A Lick spectrum taken on 2009 October 25 shows this galaxy to have strong nebular emission lines ( $\mathrm{H} \alpha, \mathrm{H} \beta, \mathrm{O}$ III, O II) and be located at a heliocentric redshift $z_{\text {hel }}=0.0371 \pm 0.0002$ (well within the Hubble flow), consistent with the estimated SN redshift. At the position of the $\mathrm{SN}, z_{\text {hel }}=0.0371$ corresponds to $z=0.0359$ in the cosmic microwave background (CMB) rest-frame $\left(z_{\mathrm{cmb}}\right)$ or a distance modulus of $35.99 \pm 0.06$ (the error assumes a host galaxy peculiar velocity uncertainty of $300 \mathrm{~km} \mathrm{~s}^{-1}$ ). This would give a projected physical separation between PTF 09dav and the center of this galaxy of $40.6 \mathrm{kpc}$.

There is no evidence for a host galaxy at the position of the SN. Using 129 "DeepSky"15 (Nugent et al. 2009) images taken with

$\overline{15}$ http://supernova.lbl.gov/ nugent/deepsky.html 


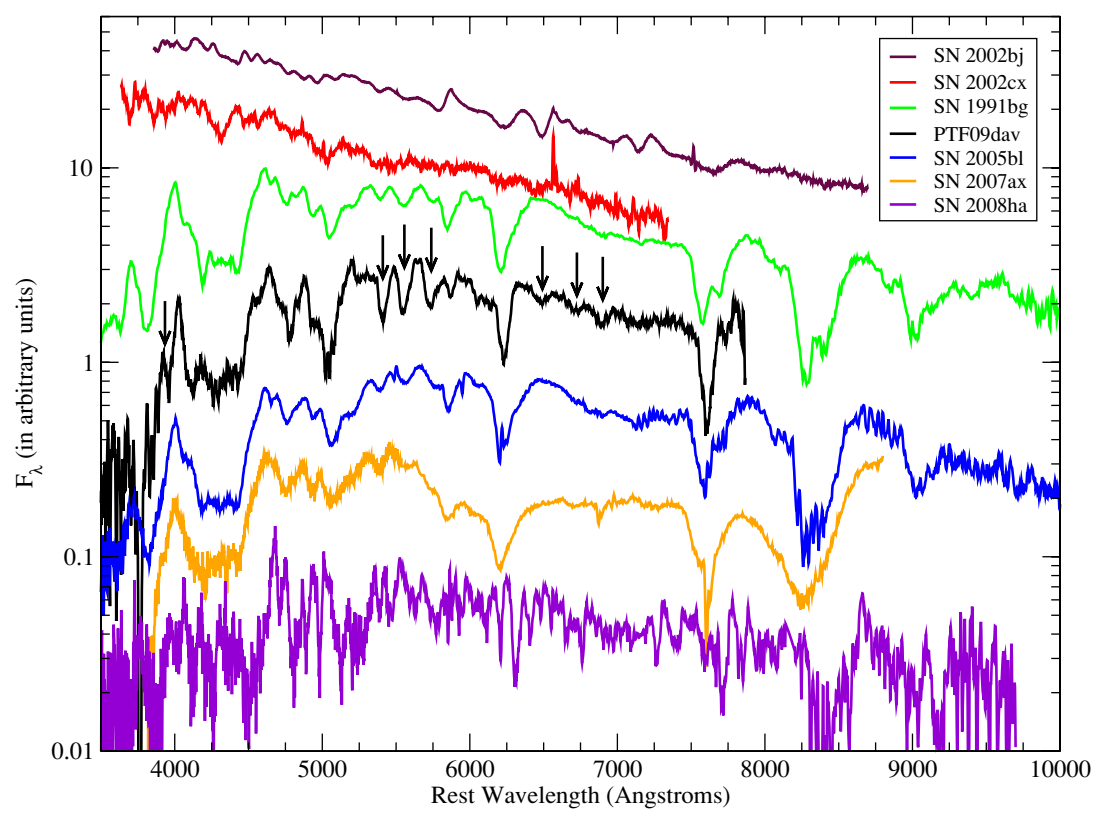

Figure 2. Comparison of the +4.9 day WHT spectrum of PTF 09dav with a selection of other subluminous events. From top to bottom: SN2002bj (Poznanski et al 2010), SN2002cx (Li et al. 2003), SN1991bg (Filippenko et al. 1992), PTF 09dav, SN2005bl (Taubenberger et al. 2008), SN2007ax (Kasliwal et al. 2008), and SN2008ha (Foley et al. 2009). For each event the spectrum nearest to +4.9 days is shown. All spectra have been deredshifted, and additionally the spectra of SN1991bg and SN2005bl have been shifted by $-3000 \mathrm{~km} \mathrm{~s}^{-1}$ to account for the velocity difference with PTF 09dav. The vertical arrows highlight the principal differences between PTF 09dav and other subluminous SNe Ia (see Figure 3 for the evolution of these features in later spectra).

(A color version of this figure is available in the online journal.)

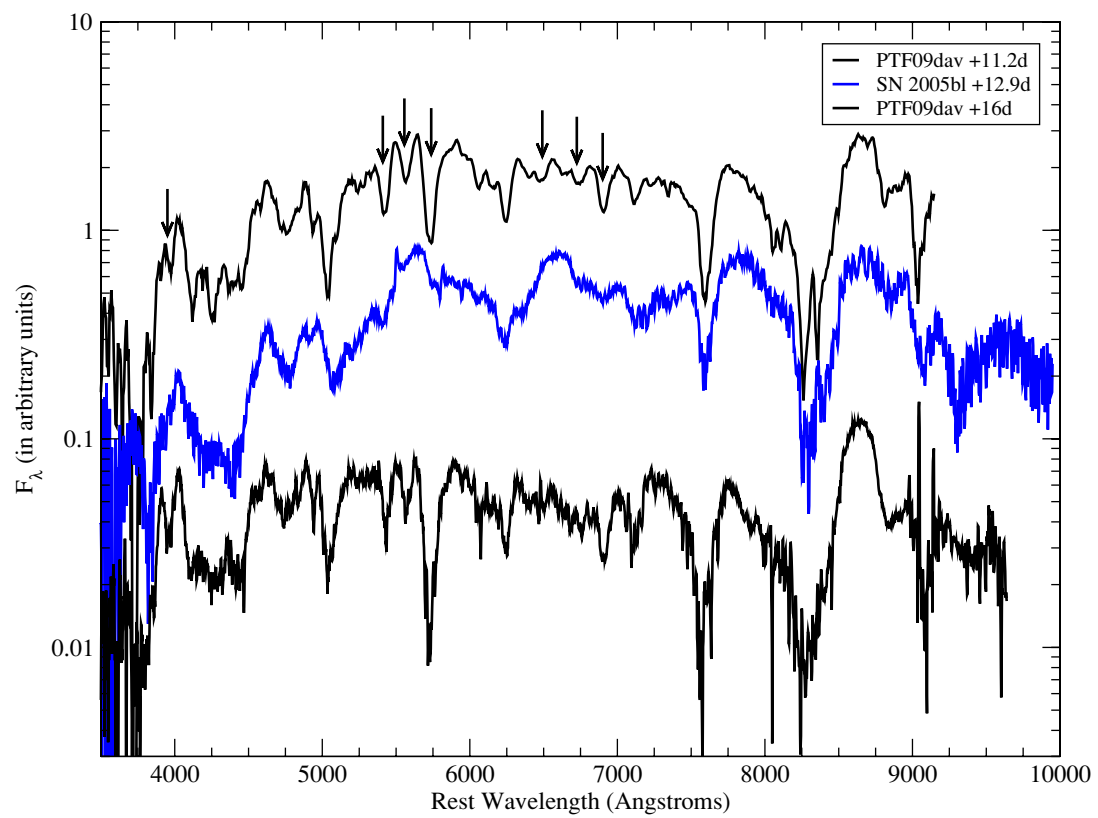

Figure 3. +11.2 day and +16 day spectra of PTF 09dav compared to SN2005bl at +12.9 days. SN2005bl has been shifted by $-3000 \mathrm{~km} \mathrm{~s}^{-1}$ to account for the velocity difference with PTF 09dav. The vertical arrows mark the same features as in Figure 2.

(A color version of this figure is available in the online journal.)

the Palomar-QUEST survey over of the field of PTF 09dav, we have constructed a deep RG610 image (a longpass $r+i+z$ filter). Using a 4" diameter aperture at the position of the $\mathrm{SN}$, we detect no host galaxy with a $3 \sigma$ limiting magnitude of $m=23.2$ in the RG610 or $M=-12.8$ at $z=0.0359$. Such a faint absolute magnitude would be lower than any detected host galaxy of a core collapse SN found by PTF (Arcavi et al. 2010). Deeper Keck imaging at the position of PTF 09dav is underway (M. M. Kasliwal et al. 2011, in preparation).

\subsection{Light-curve Data}

Photometric monitoring in gri filters commenced using the robotic Palomar 60 in (P60) telescope (Cenko et al. 2006) on 2009 August 15.3 and on the robotic Faulkes Telescope North (FTN) on 2009 August 18.4, complementing the rolling PTF search on the Samuel Oschin 48 inch telescope (P48) in $R$ band. The resulting multi-color light curve of PTF 09dav is shown in Figure 4. As PTF 09dav has no detected host galaxy 

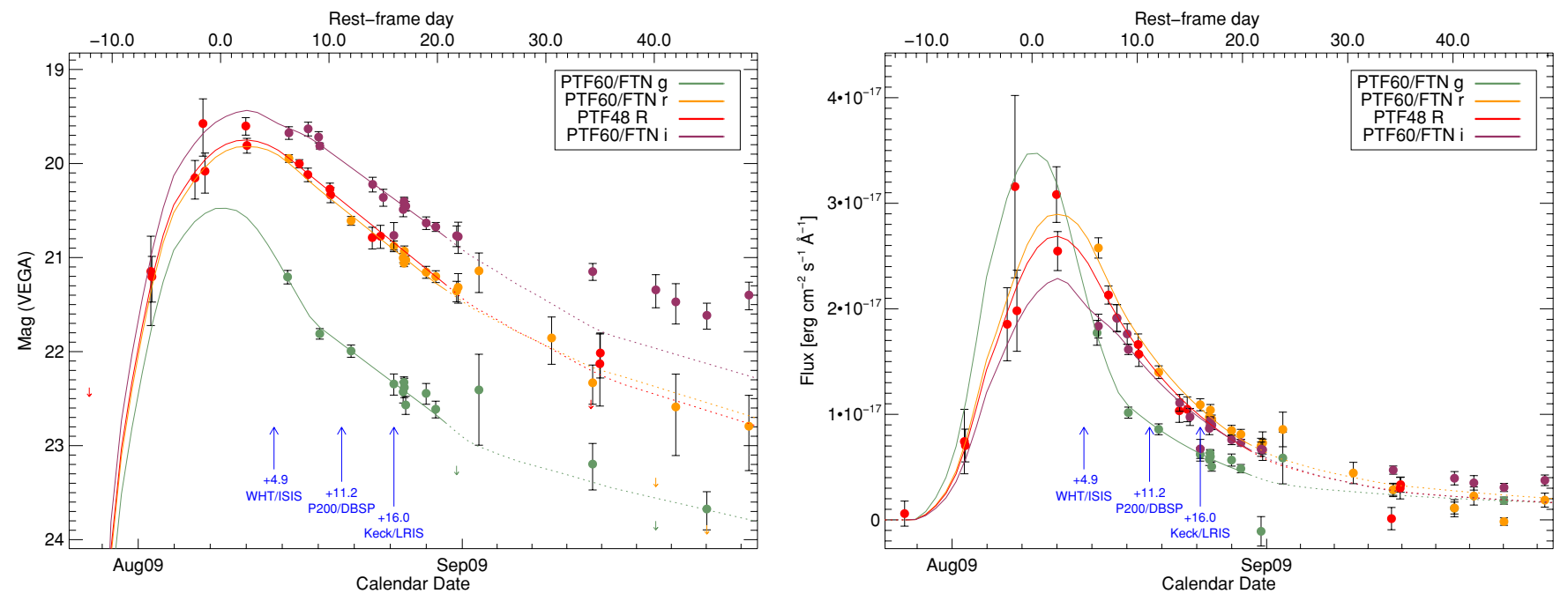

Figure 4. Observed light curve in apparent magnitude space (left) and flux space (right) for PTF 09dav in P48 $R$ and P60/FTN $g, r$, $i$. For clarity, the FTN and P60 data are denoted by the same symbol (but the correct filter responses are used in the light curve fit). The overlaid lines are the best-fitting SiFTO light curve using a subluminous SN Ia spectral template, with an arbitrary flux-scaling in each filter, but a common "stretch" factor and time of maximum light. Only datapoints up to +20 days are used in the fit; the template lines are dotted after this phase and show the predicted evolution at later phases according to the template. Maximum light occurs at an MJD of 55051.9. The arrows mark the epochs (relative to maximum light in the rest-frame $B$ band) of the three photospheric spectra in Figure 6. The reduced $\chi^{2}$ of the fit is 1.02, and in $g R r$ the light curve decay is completely consistent with that of a stretched version of SN1991bg-like SN Ia. Note that at phases later than +30 days, there is excess $i$ flux compared to the simple template prediction.

at the position of the $\mathrm{SN}$, no subtraction of a reference image is required, simplifying the light curve measurement. We measure the SN photometry using a point-spread function (PSF) fitting method. In each image frame, the PSF is determined from nearby field stars, and this average PSF is then fit at the position of the SN event weighting each pixel according to Poisson statistics, yielding an SN flux and flux error.

We perform a flux calibration to the Sloan Digital Sky Survey (SDSS) photometric system, close to the AB system (Oke \& Gunn 1983), using P48 and P60 observations of SDSS fields made on the same night as observations of PTF 09dav. We use an average calibration from photometric nights in 2009 August and September, which provides calibrated tertiary standards in the field of PTF 09dav. This calibration is then transferred to other epochs and to the FTN observations, using aperture photometry of these tertiary standards to determine an relative flux-scaling factor for each epoch, typically measured to $<1 \%$. In the P60/FTN $g, r$, and $i$ filters the color terms to the SDSS system are very small. The P48 color term is larger, and we include color, extinction, and color-extinction terms. The rms of the color term fits is $0.02-0.03 \mathrm{mag}$ (with a color term in $r-i$ of $\sim 0.22 \mathrm{mag}$ ), although we present magnitudes in the natural P48 system and do not apply this color term to our photometry. Finally, we fit the same PSF used for the SN flux measurement to the calibrating field stars, and apply a correction to go from the aperture in which the flux calibration is determined, to the PSF fit in which the SN is measured.

Although we could perform our entire analysis using magnitudes defined in the AB system, rest-frame SN Ia properties in the literature are typically given in the Vega system. For ease of comparison to previous work, we convert our calibrated light curves into this system when performing the light curve analysis, and all quoted magnitudes are given in the Vega system. The offsets from Vega $\left(m_{\mathrm{vega}}\right)$ to $\mathrm{AB}\left(m_{\mathrm{AB}}\right)$ magnitudes are -0.12 , $0.13,0.19$, and 0.35 for the $g, r, R$, and $i$ filters, respectively ( $m_{\text {vega }}=m_{\mathrm{AB}}-$ offset). Our light curve data can be found in Table 1, presented in counts rather than magnitudes to preserve information on non-detections.

\section{LIGHT CURVE ANALYSIS}

\subsection{Method}

Estimating the peak luminosity of $\mathrm{SNe}$ in a given bandpass requires an interpolation between observed data points at the time of maximum light, followed by a $k$-correction back to some standard rest-frame filter of interest. The interpolation step is best performed using a smooth light curve template in the observed filter which is believed to represent the photometric evolution of the $\mathrm{SN}$; the $k$-correction requires a time series spectral energy distribution (SED; e.g., Nugent et al. 2002; Hsiao et al. 2007). To parametrize the light curve of PTF 09dav, we fit a time series SED to the observed photometry using the SiFTO light curve fitter (Conley et al. 2008) developed for SN Ia cosmology studies. We replace the standard SN Ia spectral template $^{16}$ with one based on the subluminous SN Ia events SN1991bg (Filippenko et al. 1992; Leibundgut et al. 1993) and SN1999by (Garnavich et al. 2004). Ideally, one would use an SED template which exactly matches the spectral class of the object under study. For PTF 09dav, the first object of its type, no such template exists, so we use our subluminous template which has many common spectral features and similar broadband colors.

SiFTO works in flux space, manipulating a model of the SED and synthesizing an observer-frame light curve from a given spectral time series in a set of filters at a given redshift, allowing an arbitrary normalization in each observed filter (i.e., the absolute colors of the template being fit are not important and do not influence the fit). The time-axis of the template is adjusted by a dimensionless relative "stretch" ( $s$ ) factor (where the input template is defined to have $s=1$ ) to fit the data. Once the observer-frame SiFTO fit is complete, it can be used to estimate rest-frame magnitudes in any given set of filters, provided there is equivalent observer-frame filter coverage and at any epoch. This is performed by adjusting the template SED at the required epoch to have the correct observed

\footnotetext{
16 http://supernova.lbl.gov/ nugent/nugent_templates.html
} 
Table 1

Photometry for PTF 09dav (Photometry is Listed for a Common Zeropoint of 30a)

\begin{tabular}{|c|c|c|c|c|c|c|c|c|c|}
\hline \multirow[t]{2}{*}{$\mathrm{MJD}^{\mathrm{b}}$} & \multicolumn{2}{|c|}{$g$} & \multicolumn{2}{|c|}{$r$} & \multicolumn{2}{|c|}{$R$} & \multicolumn{2}{|c|}{$i$} & \multirow[t]{2}{*}{ Telescope } \\
\hline & Counts & Error & Counts & Error & Counts & Error & Counts & Error & \\
\hline 55039.32 & $\ldots$ & $\ldots$ & $\ldots$ & $\ldots$ & 278.5 & 557.0 & $\ldots$ & $\ldots$ & P48 \\
\hline 55045.21 & $\ldots$ & $\ldots$ & $\ldots$ & $\ldots$ & 3476.9 & 1430.7 & $\ldots$ & $\ldots$ & P48 \\
\hline 55045.31 & $\ldots$ & $\ldots$ & $\ldots$ & $\ldots$ & 3300.0 & 726.0 & $\ldots$ & $\ldots$ & P48 \\
\hline 55049.43 & $\ldots$ & $\ldots$ & $\ldots$ & $\ldots$ & 8683.0 & 1621.0 & $\ldots$ & $\ldots$ & P48 \\
\hline 55050.20 & $\ldots$ & $\ldots$ & $\ldots$ & $\ldots$ & 14791.5 & 4045.6 & $\ldots$ & $\ldots$ & $\mathrm{P} 48$ \\
\hline 55050.39 & $\ldots$ & $\ldots$ & $\ldots$ & $\ldots$ & 9281.0 & 1799.0 & $\ldots$ & $\ldots$ & P48 \\
\hline 55054.29 & $\ldots$ & $\ldots$ & $\ldots$ & $\ldots$ & 14438.4 & 1234.6 & $\ldots$ & $\ldots$ & P48 \\
\hline 55054.40 & $\ldots$ & $\ldots$ & $\ldots$ & $\ldots$ & 11930.8 & 865.1 & $\ldots$ & $\ldots$ & P48 \\
\hline 55058.29 & 3292.3 & 244.1 & $\ldots$ & $\ldots$ & $\ldots$ & $\ldots$ & $\ldots$ & $\ldots$ & P60 \\
\hline 55058.42 & $\ldots$ & $\ldots$ & 10508.5 & 346.9 & $\ldots$ & $\ldots$ & $\ldots$ & $\ldots$ & P60 \\
\hline 55058.42 & $\ldots$ & $\ldots$ & $\ldots$ & $\ldots$ & $\ldots$ & $\ldots$ & 13501.6 & 601.9 & P60 \\
\hline 55059.41 & $\ldots$ & $\ldots$ & $\ldots$ & $\ldots$ & 9980.0 & 403.0 & $\ldots$ & $\ldots$ & P48 \\
\hline 55060.23 & $\ldots$ & $\ldots$ & $\ldots$ & $\ldots$ & 8964.8 & 594.3 & $\ldots$ & $\ldots$ & P48 \\
\hline 55060.25 & $\ldots$ & $\ldots$ & $\ldots$ & $\ldots$ & $\ldots$ & $\ldots$ & 14045.7 & 681.9 & P60 \\
\hline 55061.26 & $\ldots$ & $\ldots$ & $\ldots$ & $\ldots$ & $\ldots$ & $\ldots$ & 12955.2 & 512.7 & P60 \\
\hline 55061.37 & 1887.6 & 109.0 & $\ldots$ & $\ldots$ & $\ldots$ & $\ldots$ & $\ldots$ & $\ldots$ & FTN \\
\hline 55061.37 & $\ldots$ & $\ldots$ & $\ldots$ & $\ldots$ & $\ldots$ & $\ldots$ & 11874.5 & 254.2 & FTN \\
\hline 55062.34 & $\ldots$ & $\ldots$ & $\ldots$ & $\ldots$ & 7782.0 & 471.0 & $\ldots$ & $\ldots$ & $\mathrm{P} 48$ \\
\hline 55062.40 & $\ldots$ & $\ldots$ & $\ldots$ & $\ldots$ & 7353.0 & 542.0 & $\ldots$ & $\ldots$ & P48 \\
\hline 55064.36 & 1594.8 & 107.0 & $\ldots$ & $\ldots$ & $\ldots$ & $\ldots$ & $\ldots$ & $\ldots$ & P60 \\
\hline 55064.36 & $\ldots$ & $\ldots$ & 5706.7 & 217.3 & $\ldots$ & $\ldots$ & $\ldots$ & $\ldots$ & P60 \\
\hline 55066.39 & $\ldots$ & $\ldots$ & $\ldots$ & $\ldots$ & 4843.1 & 514.2 & $\ldots$ & $\ldots$ & P48 \\
\hline 55066.43 & $\ldots$ & $\ldots$ & $\ldots$ & $\ldots$ & $\ldots$ & $\ldots$ & 8154.8 & 423.1 & P60 \\
\hline 55067.17 & $\ldots$ & $\ldots$ & $\ldots$ & $\ldots$ & 4906.7 & 551.5 & $\ldots$ & $\ldots$ & $\mathrm{P} 48$ \\
\hline 55067.45 & $\ldots$ & $\ldots$ & $\ldots$ & $\ldots$ & $\ldots$ & $\ldots$ & 7173.5 & 429.3 & P60 \\
\hline 55068.45 & 1154.2 & 133.2 & $\ldots$ & $\ldots$ & $\ldots$ & $\ldots$ & $\ldots$ & $\ldots$ & P60 \\
\hline 55068.45 & $\ldots$ & $\ldots$ & 4449.1 & 206.4 & $\ldots$ & $\ldots$ & $\ldots$ & $\ldots$ & P60 \\
\hline 55068.46 & $\ldots$ & $\ldots$ & $\ldots$ & $\ldots$ & $\ldots$ & $\ldots$ & 4945.3 & 480.3 & P60 \\
\hline 55069.35 & 1068.0 & 122.9 & $\ldots$ & $\ldots$ & $\ldots$ & $\ldots$ & $\ldots$ & $\ldots$ & FTN \\
\hline 55069.35 & $\ldots$ & $\ldots$ & 3964.3 & 156.1 & $\ldots$ & $\ldots$ & $\ldots$ & $\ldots$ & FTN \\
\hline 55069.35 & $\ldots$ & $\ldots$ & $\ldots$ & $\ldots$ & $\ldots$ & $\ldots$ & 6374.3 & 314.6 & FTN \\
\hline 55069.42 & 1172.7 & 72.6 & $\ldots$ & $\ldots$ & $\ldots$ & $\ldots$ & $\ldots$ & $\ldots$ & FTN \\
\hline 55069.43 & $\ldots$ & $\ldots$ & 3766.2 & 110.0 & $\ldots$ & $\ldots$ & $\ldots$ & $\ldots$ & FTN \\
\hline 55069.43 & $\ldots$ & $\ldots$ & $\ldots$ & $\ldots$ & $\ldots$ & $\ldots$ & 6898.8 & 209.0 & FTN \\
\hline 55069.46 & 1115.6 & 117.5 & $\ldots$ & $\ldots$ & $\ldots$ & $\ldots$ & $\ldots$ & $\ldots$ & P60 \\
\hline 55069.46 & $\ldots$ & $\ldots$ & 4243.1 & 191.0 & $\ldots$ & $\ldots$ & $\ldots$ & $\ldots$ & P60 \\
\hline 55069.46 & $\ldots$ & $\ldots$ & $\ldots$ & $\ldots$ & $\ldots$ & $\ldots$ & 6702.6 & 292.5 & P60 \\
\hline 55069.59 & 939.6 & 93.0 & $\ldots$ & $\ldots$ & $\ldots$ & $\ldots$ & $\ldots$ & $\ldots$ & FTN \\
\hline 55069.59 & $\ldots$ & $\ldots$ & 3893.7 & 138.7 & $\ldots$ & $\ldots$ & $\ldots$ & $\ldots$ & FTN \\
\hline 55069.60 & $\ldots$ & $\ldots$ & $\ldots$ & $\ldots$ & $\ldots$ & $\ldots$ & 6591.3 & 220.9 & FTN \\
\hline 55071.54 & 1052.9 & 119.4 & $\ldots$ & $\ldots$ & $\ldots$ & $\ldots$ & $\ldots$ & $\ldots$ & FTN \\
\hline 55071.55 & $\ldots$ & $\ldots$ & 3450.9 & 178.2 & $\ldots$ & $\ldots$ & $\ldots$ & $\ldots$ & FTN \\
\hline 55071.55 & $\ldots$ & $\ldots$ & $\ldots$ & $\ldots$ & $\ldots$ & $\ldots$ & 5585.9 & 248.2 & FTN \\
\hline 55072.46 & 901.7 & 82.6 & $\ldots$ & $\ldots$ & $\ldots$ & $\ldots$ & $\ldots$ & $\ldots$ & FTN \\
\hline 55072.46 & $\ldots$ & $\ldots$ & 3307.5 & 169.4 & $\ldots$ & $\ldots$ & $\ldots$ & $\ldots$ & FTN \\
\hline 55072.47 & $\ldots$ & $\ldots$ & $\ldots$ & $\ldots$ & $\ldots$ & $\ldots$ & 5377.1 & 171.4 & FTN \\
\hline 55074.45 & -201.6 & 289.7 & $\ldots$ & $\ldots$ & $\ldots$ & $\ldots$ & $\ldots$ & $\ldots$ & FTN \\
\hline 55074.46 & $\ldots$ & $\ldots$ & 2868.6 & 252.2 & $\ldots$ & $\ldots$ & $\ldots$ & $\ldots$ & FTN \\
\hline 55074.46 & $\ldots$ & $\ldots$ & $\ldots$ & $\ldots$ & $\ldots$ & $\ldots$ & 4928.4 & 359.2 & FTN \\
\hline 55074.60 & $\ldots$ & $\ldots$ & 2974.2 & 378.5 & $\ldots$ & $\ldots$ & $\ldots$ & $\ldots$ & FTN \\
\hline 55074.61 & $\ldots$ & $\ldots$ & $\ldots$ & $\ldots$ & $\ldots$ & $\ldots$ & 4887.1 & 536.0 & FTN \\
\hline 55076.59 & 1089.1 & 509.4 & $\ldots$ & $\ldots$ & $\ldots$ & $\ldots$ & $\ldots$ & $\ldots$ & FTN \\
\hline 55076.60 & $\ldots$ & $\ldots$ & 3494.6 & 591.3 & $\ldots$ & $\ldots$ & $\ldots$ & $\ldots$ & FTN \\
\hline 55083.54 & $\ldots$ & $\ldots$ & 1811.6 & 364.3 & $\ldots$ & $\ldots$ & $\ldots$ & $\ldots$ & FTN \\
\hline 55087.31 & $\ldots$ & $\ldots$ & $\ldots$ & $\ldots$ & 56.0 & 494.0 & $\ldots$ & $\ldots$ & $\mathrm{P} 48$ \\
\hline 55087.47 & 526.7 & 131.9 & $\ldots$ & $\ldots$ & $\ldots$ & $\ldots$ & $\ldots$ & $\ldots$ & FTN \\
\hline 55087.47 & $\ldots$ & $\ldots$ & 1167.9 & 196.1 & $\ldots$ & $\ldots$ & $\ldots$ & $\ldots$ & FTN \\
\hline 55087.48 & $\ldots$ & $\ldots$ & $\ldots$ & $\ldots$ & $\ldots$ & $\ldots$ & 3470.4 & 206.9 & FTN \\
\hline 55088.16 & $\ldots$ & $\ldots$ & $\ldots$ & $\ldots$ & 1406.8 & 477.0 & $\ldots$ & $\ldots$ & $\mathrm{P} 48$ \\
\hline 55088.21 & $\ldots$ & $\ldots$ & $\ldots$ & $\ldots$ & 1563.0 & 339.0 & $\ldots$ & $\ldots$ & $\mathrm{P} 48$ \\
\hline 55093.50 & 205.2 & 168.6 & $\ldots$ & $\ldots$ & $\ldots$ & $\ldots$ & $\ldots$ & $\ldots$ & FTN \\
\hline 55093.51 & $\ldots$ & $\ldots$ & 457.8 & 203.6 & $\ldots$ & $\ldots$ & $\ldots$ & $\ldots$ & FTN \\
\hline 55093.51 & $\ldots$ & $\ldots$ & $\ldots$ & $\ldots$ & $\ldots$ & $\ldots$ & 2900.7 & 336.5 & FTN \\
\hline 55095.42 & $\ldots$ & $\ldots$ & 921.9 & 308.9 & $\ldots$ & $\ldots$ & $\ldots$ & $\ldots$ & P60 \\
\hline
\end{tabular}


Table 1

(Continued)

\begin{tabular}{|c|c|c|c|c|c|c|c|c|c|}
\hline \multirow[t]{2}{*}{$\mathrm{MJD}^{\mathrm{b}}$} & \multicolumn{2}{|c|}{$g$} & \multicolumn{2}{|c|}{$r$} & \multicolumn{2}{|c|}{$R$} & \multicolumn{2}{|c|}{$i$} & \multirow[t]{2}{*}{ Telescope } \\
\hline & Counts & Error & Counts & Error & Counts & Error & Counts & Error & \\
\hline 55095.42 & & & $\ldots$ & $\ldots$ & $\ldots$ & $\ldots$ & 2579.6 & 363.5 & $\mathrm{P} 60$ \\
\hline 55098.39 & 339.2 & 70.1 & $\ldots$ & $\ldots$ & $\ldots$ & $\ldots$ & $\ldots$ & $\ldots$ & FTN \\
\hline 55098.39 & $\ldots$ & $\ldots$ & -66.3 & 128.0 & $\ldots$ & $\ldots$ & $\ldots$ & $\ldots$ & FTN \\
\hline 55098.40 & $\ldots$ & $\ldots$ & $\ldots$ & $\ldots$ & $\ldots$ & $\ldots$ & 2260.3 & 206.4 & FTN \\
\hline 55102.42 & $\ldots$ & $\ldots$ & 763.3 & 238.0 & $\ldots$ & $\ldots$ & $\ldots$ & $\ldots$ & FTN \\
\hline 55102.42 & $\ldots$ & $\ldots$ & $\ldots$ & $\ldots$ & $\ldots$ & $\ldots$ & 2757.1 & 268.0 & FTN \\
\hline 55123.25 & $\ldots$ & $\ldots$ & $\ldots$ & $\ldots$ & 2179.4 & 584.9 & $\ldots$ & $\ldots$ & $\mathrm{P} 48$ \\
\hline 55138.20 & $\ldots$ & $\ldots$ & $\ldots$ & $\ldots$ & -659.2 & 1475.4 & $\ldots$ & $\ldots$ & P48 \\
\hline 55142.19 & $\ldots$ & $\ldots$ & $\ldots$ & $\ldots$ & 272.3 & 704.7 & $\ldots$ & $\ldots$ & P48 \\
\hline
\end{tabular}

Notes.

${ }^{\text {a }}$ Convert (positive) counts, $c$, to Vega magnitudes, $m$, using $m=-2.5 \log (c)+30$.

${ }^{\mathrm{b}}$ Modified Julian Date; MJD = JD - 2400000.5.

colors from the SIFTO fit, correcting for extinction along the line of sight in the Milky Way, de-redshifting, and integrating the resultant SED through the required filters. This process is essentially a cross-filter $k$-correction, with the advantage that all the observed data contribute to the SED shape used. As an illustration, the size of the $k$-correction for an SN1991bg-like SN Ia to go from observer $g(r)$ at $z=0.0371$ to rest-frame $B(V)$ is $\sim-0.16 \mathrm{mag}(\sim-0.24 \mathrm{mag})$ at maximum light and $\sim-0.28 \mathrm{mag}(\sim-0.48 \mathrm{mag})$ at 10 days after maximum.

The function used to adjust the template SED can either be an interpolating spline (in which case a perfect match in color is attained) or, if the reason for the physical difference in the colors between the SN and the template is understood, a function such as a standard dust law (e.g., Cardelli et al. 1989) or other wavelength-dependent color law (e.g., Guy et al. 2007). The latter case is more useful when extrapolating beyond the wavelength range of the observed filters, although it gives a poorer match between observed and fit colors.

\subsection{Fit Results}

Our SiFTO light curve fit is in Figure 4. The fit is good-the reduced $\chi^{2}$ of the fit is 1.02 for 40 degrees of freedom (i.e., $\chi^{2}=40.8 / 40$ ) - using a standard SN Ia template in SiFTO gives a substantially worse reduced $\chi^{2}$ of 4.3. Maximum light in the rest-frame $B$ band was on 2009 August $08.9 \pm 0.3$ days (or an MJD of 55051.9), i.e., the SN was discovered some 3 days after maximum light. Interestingly, although the fit to the data around maximum light is excellent, at phases beyond $\sim+30$ days there appears to be significant excess $i$-band flux in the SN compared to the simple prediction of the template with the $i$-band light curve appearing to plateau, while the decay in the other filters continues to follow the subluminous template. This could be due to emerging nebular emission from the [Ca II] doublet at $\lambda 7291$ and $\lambda 7323$, with stronger lines at that phase than in our SN1991bg-like subluminous template, and more similar to later time spectra of calcium-rich $\mathrm{SNe}$ (Perets et al. 2010).

As PTF 09dav has no detected host galaxy at the SN position, we can cross-check our photometric calibration using photometry synthesized from our WHT spectrum. At the time of the WHT spectrum ( +4.9 days), SiFTO gives a light curve color of $g-R=1.17 \pm 0.05$, compared to a spectral color from the WHT spectrum of $g-R=1.26$. Given the uncertainties in fluxcalibrating long-slit spectra (e.g., differential slit losses which will redden the SED) as well as in the P48 $R$ and P60 $g$ filter responses used for synthetic photometry, this is an excellent level of agreement suggesting an accurate photometric flux calibration. A similar agreement is found for the later P200 spectrum.

Our reported magnitudes are in the Vega system, taking our $B V R$ filter responses from Bessell (1990) and correcting for Milky Way extinction assuming $R_{V}=3.1$ and a color excess of $E(B-V)_{\mathrm{mw}}=0.044$ (Schlegel et al. 1998) with a Cardelli et al. (1989) extinction law. We measure peak restframe absolute magnitudes at the time of $B$-band maximum light of $M_{B}=-15.44 \pm 0.05, M_{V}=-16.01 \pm 0.05$, and $M_{R}=$ $-16.26 \pm 0.05$ (errors are statistical uncertainties propagated through the light curve fit only) using a CCM law in SiFTO to match the template to the observed fit colors; we adopt these numbers throughout this paper. For comparison, using a spline in SIFTO in place of the CCM law gives $M_{B}=-15.54 \pm 0.05$, $M_{V}=-15.98 \pm 0.09$, and $M_{R}=-16.24 \pm 0.05$.

One potential concern in the light curve fit is that the P60/ FTN $g$ coverage, corresponding to rest-frame $B$, did not start until $\simeq 6$ days after maximum light—-and so the $B$-band (and $V$-band) maximum light estimate is an extrapolation (based on the light curves synthesized from the SED template) rather than an interpolation as with the P48 $R$ band. Our key assumption is that the light curve of PTF 09dav is well represented by a "stretched" version of our subluminous spectral template. While this is clearly true in the P48 $R$ data where the fit before and after maximum light is robust, in $g$ the data are not sufficient to test this assumption.

\subsection{Comparison to Other Events}

A comparison of PTF 09dav to other SN1991bg-like subluminous SN Ia events can be found in Table 2 and Figure 5. With no commonly accepted definition for what constitutes an SN1991bg-like event, we fit a combination of events matching the cool classification from Branch et al. (2009), events with $\Delta M_{15}(B)>1.8$ from Taubenberger et al. (2008) and (Hicken et al. 2009), and other individual events from the literature (e.g., Kasliwal et al. 2008). We fit all the public SN Ia photometry with SiFTO and the same subluminous template to ensure a consistent comparison. All magnitudes are corrected for Milky Way extinction, and we have assumed distance moduli either calculated from $z_{\mathrm{cmb}}$ when $z_{\mathrm{cmb}} \geqslant 0.01$ or taken from a redshiftindependent estimate (together with an uncertainty) from the NASA/IPAC Extragalactic Database $\left(\mathrm{NED}^{17}\right)$ in other cases.

\footnotetext{
17 http://nedwww.ipac.caltech.edu/
} 
Table 2

Photometric Properties of PTF 09dav Compared to 1991bg-like SNe Ia

\begin{tabular}{|c|c|c|c|c|c|}
\hline Name & $M_{B}{ }^{\mathrm{a}}$ & $(B-V)_{\max }$ & "stretch"b & $L_{\text {bol }}{ }^{\mathrm{c}}\left(\times 10^{42} \mathrm{erg} \mathrm{s}^{-1}\right)$ & $M_{\mathrm{Ni}}^{\mathrm{d}}\left(M_{\odot}\right)$ \\
\hline PTF09dav & $-15.44 \pm 0.08$ & $0.56 \pm 0.07$ & $0.86 \pm 0.02$ & $0.56 \pm 0.04$ & $0.019 \pm 0.003$ \\
\hline $\operatorname{sn} 2007 \mathrm{ax}$ & $-16.15 \pm 0.17$ & $0.62 \pm 0.03$ & $0.82 \pm 0.02$ & $1.13 \pm 0.18$ & $0.038 \pm 0.008$ \\
\hline sn2007al & $-16.41 \pm 0.17$ & $0.65 \pm 0.03$ & $0.81 \pm 0.03$ & $1.50 \pm 0.23$ & $0.050 \pm 0.010$ \\
\hline sn1991bg & $-16.71 \pm 0.20$ & $0.66 \pm 0.03$ & $0.83 \pm 0.04$ & $2.01 \pm 0.37$ & $0.068 \pm 0.016$ \\
\hline $\operatorname{sn} 2005 \mathrm{bl}$ & $-16.72 \pm 0.09$ & $0.60 \pm 0.02$ & $0.96 \pm 0.01$ & $1.89 \pm 0.15$ & $0.072 \pm 0.011$ \\
\hline sn2006je & $-16.85 \pm 0.07$ & $0.64 \pm 0.06$ & $0.93 \pm 0.09$ & $2.24 \pm 0.15$ & $0.083 \pm 0.014$ \\
\hline sn1998de & $-16.97 \pm 0.07$ & $0.51 \pm 0.03$ & $1.00 \pm 0.01$ & $2.13 \pm 0.15$ & $0.084 \pm 0.012$ \\
\hline sn2005ke & $-16.98 \pm 0.20$ & $0.57 \pm 0.02$ & $1.08 \pm 0.01$ & $2.29 \pm 0.42$ & $0.096 \pm 0.021$ \\
\hline sn1999da & $-17.03 \pm 0.02$ & $0.48 \pm 0.03$ & $0.99 \pm 0.02$ & $2.18 \pm 0.05$ & $0.085 \pm 0.011$ \\
\hline sn $2006 b z$ & $-17.16 \pm 0.08$ & $0.55 \pm 0.01$ & $0.90 \pm 0.02$ & $2.65 \pm 0.20$ & $0.096 \pm 0.014$ \\
\hline sn1997cn & $-17.16 \pm 0.13$ & $0.54 \pm 0.04$ & $0.85 \pm 0.03$ & $2.61 \pm 0.32$ & $0.090 \pm 0.016$ \\
\hline sn1999da & $-17.21 \pm 0.09$ & $0.48 \pm 0.03$ & $0.99 \pm 0.02$ & $2.59 \pm 0.22$ & $0.101 \pm 0.015$ \\
\hline sn1999by & $-17.22 \pm 0.20$ & $0.44 \pm 0.01$ & $0.99 \pm 0.01$ & $2.52 \pm 0.47$ & $0.098 \pm 0.022$ \\
\hline sn1998bp & $-17.81 \pm 0.11$ & $0.41 \pm 0.04$ & $1.01 \pm 0.06$ & $4.18 \pm 0.43$ & $0.166 \pm 0.028$ \\
\hline sn2007au & $-18.20 \pm 0.03$ & $0.20 \pm 0.04$ & $1.16 \pm 0.04$ & $5.01 \pm 0.13$ & $0.224 \pm 0.027$ \\
\hline
\end{tabular}

Notes.

${ }^{a}$ Corrected for Milky Way extinction but not for any host galaxy extinction. Assumes $H_{0}=70 \mathrm{~km} \mathrm{~s}^{-1} \mathrm{Mpc}^{-1}$.

b Relative to our subluminous SN Ia template. We emphasize that these stretches cannot be compared to stretches derived using a normal SN Ia light curve template which have a very different definition of $s=1$.

${ }^{c}$ Estimated at the peak in the bolometric light curve.

${ }^{\mathrm{d}}$ Assumes that $\tau_{r}$ for each SN is given by the SN stretch in Column 4 multiplied by the template rise time of 14 days.

We have also propagated through a $300 \mathrm{~km} \mathrm{~s}^{-1}$ peculiar velocity error for those SNe Ia in the Hubble flow. We only consider SNe with adequate photometry for a reliable light curve fit, and those with $M_{B}>-18$ at maximum light, in our analysis.

The SNe show expected trends between stretch, color, and $M_{B}$ (Figure 5) which are consistent with previous studies (Garnavich et al. 2004; Taubenberger et al. 2008; Gonzalez-Gaitan et al. 2011): fainter $\mathrm{SNe}$ are redder and with smaller values of stretch. (Note that the stretches here are measured relative to our subluminous template and are not on the same system as stretches of normal SNe Ia.) Clearly PTF 09dav is an outlier in these relations, being considerably fainter for its color and stretch than other subluminous events: PTF 09dav is $\sim 3.5 \mathrm{mag}$ underluminous compared to normal SNe Ia $\left(M_{B} \simeq-19.1\right)$, $\sim 1.5$ mag fainter than a typical subluminous $\mathrm{SN}$ Ia, and around $\sim 0.5$ mag fainter than SN2007ax, the faintest subluminous SN Ia identified (Kasliwal et al. 2008).

No correction for host extinction (if any is present) has been applied to the magnitudes of PTF 09dav (or the other SNe in Table 2), and thus host extinction will impact Figure 5. For PTF 09dav, $(B-V)_{\max }=0.57 \pm 0.08$, and when using the CCM law in SiFTO to match the subluminous template to the fit colors for PTF 09dav, a small additional $E(B-V)$ of $0.08 \pm 0.04$ is required. Though it may be tempting to interpret this at face value as a reddening for the $\mathrm{SN}$ (and derive similar corrections for the other $\mathrm{SNe}$ in Table 2), such an approach is likely to be dangerous. Generally, the color-luminosity variation in $\mathrm{SNe}$ Ia is poorly understood. There is considerable evidence for an intrinsic color-luminosity relation which is currently impossible to disentangle from the effects of dust unless the SN is particularly well observed. Thus, interpreting a red color as evidence of extinction may not be correct. A second consideration is that our subluminous template may not be appropriate for zero extinction, as it is based on real SN photometry. Therefore, we simply note that the broadband colors of PTF 09dav are similar to other subluminous SN Ia events and show no evidence for significant host galaxy extinction.
We also estimate the value of the Phillips (1993) decline rate parameter $\Delta m_{15}(B)$ (the amount in magnitudes in the rest-frame $B$ band that the $\mathrm{SN}$ declines in the 15 days following maximum light) as $\Delta m_{15}(B)=1.87 \pm 0.06$. The error is a combination of propagating the error in the stretch parameter, combined with the uncertainty in peak $B$-band brightness. Numerous studies have fit the relation between $\Delta m_{15}(B)$ and $M_{B}$ for subluminous SNe Ia (e.g., Garnavich et al. 2004; Taubenberger et al. 2008). According to the relation of Taubenberger et al. (2008), our $\Delta m_{15}(B)=1.87$ should correspond to a peak $B$ absolute magnitude of -17.44 , nearly two magnitudes brighter than we observe $\left(M_{B} \simeq-15.5\right.$ corresponds to $\Delta m_{15}(B) \simeq 2.15$ according to the Taubenberger relation). This is consistent with PTF 09dav as an outlier in Figure 5. However, $\Delta m_{15}(B)$, unlike stretch, can be misleading in these comparisons. In the fainter examples of subluminous SNe Ia, the transition from the fast initial decline to the slower late-time decline occurs before -15 days, which distorts the relation (e.g., Kasliwal et al. 2008).

\subsection{Rise Time and Energetics}

The subluminous template we used in the SiFTO fit has a rise time $\left(\tau_{r}\right)$ from explosion of 13.0 days to $B$-band maximum light and 14.0 days to bolometric maximum light. PTF 09dav has a light curve "stretch" factor of $0.86 \pm 0.02$ compared to this template, giving a rise time to bolometric peak of $12.0 \pm 0.3$ days (statistical error only). We also estimate the rise time by fitting the P48 $R$ data in the early rise-time region using the analytical equation $f(t)=\alpha\left(\tau+\tau_{r}\right)^{2}$ (Riess et al. 1999). Here, $\tau=t /(1+z)$, where $t$ is the observed time and $\alpha$ is a normalizing constant. We find rise times of $12.0 \pm 2.9$ when fitting the first two epochs (including the first non-detection) and $14.5 \pm 1.1$ when including the next epoch. These are consistent with our estimates from the template light curve fit. The latter measure includes data from near-maximum light, where the assumption of the simple rise-time model may no longer hold, and should be considered a strong upper limit.

Using our light curve fits, we can also estimate the bolometric luminosity, $L_{\text {bol }}$, of PTF 09dav. Estimating the bolometric 


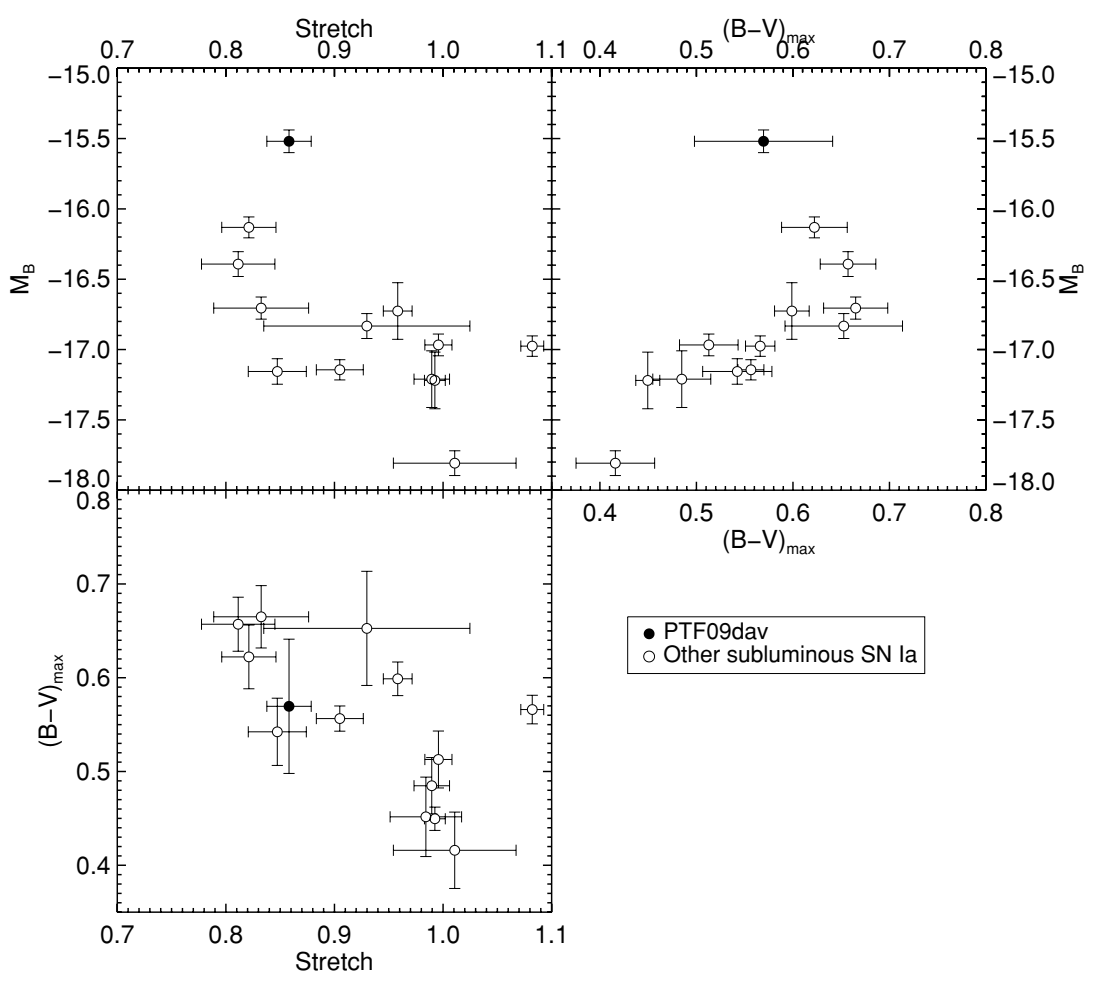

Figure 5. Comparison of the photometric properties of PTF 09dav and other $1991 \mathrm{bg}$-like subluminous SNe Ia. Top left: stretch vs. $M_{B}$, top right: $M_{B}$ vs. color $\left((B-V)_{\max }\right)$, and lower left: stretch vs. $(B-V)_{\max }$. Here, the stretch is measured relative to our subluminous template and is not on the same system as those used in cosmological studies. All magnitudes are corrected for Milky Way extinction. In all panels, PTF 09dav is shown as the filled circle, and the other subluminous SNe Ia is denoted by the open circles. The data are given in Table 2 .

luminosity is never straightforward as full ultraviolet through infrared (or UVOIR) light curves are rarely observed. Instead, we measure the luminosity in a given band together with the colors from our light curve fits and use the template to derive a bolometric luminosity estimate (effectively a bolometric correction). Such techniques work well for SNe Ia events near maximum light as the peak of the luminosity output is in the optical. Again, as with the light curve fit, we use the template spectrum to calculate our bolometric light curve. In principle we could use the observed spectra, but the lack of an observation at maximum light, and the lack of $\lambda>7700$ data in the spectrum nearest maximum, makes this difficult. Our technique broadly matches that of Howell et al. (2009). We take the template spectrum on the day of maximum light, adjust it using a CCM law to have the correct color, normalize to the peak $B$-band absolute magnitude, and integrate the resulting spectrum. The peak bolometric luminosity (occurring $\simeq 1$ day after $B$-band peak) is estimated to be $L_{\text {bol }}^{B}=5.6 \pm 0.4 \times 10^{41} \mathrm{erg} \mathrm{s}^{-1}$ (Table 2). The implicit bolometric correction, defined as $\mathrm{BC}=M_{\mathrm{bol}}-$ $M_{V}$, where $M_{\text {bol }}$ is the bolometric magnitude, is $\simeq 0.2 \mathrm{mag}$, comparable to other subluminous events SN Ia events (Contardo et al. 2000).

The ejecta mass $M_{\text {ej }}$ and kinetic energy $E_{\text {kin }}$ of PTF 09dav can be estimated from its rise time and ejecta velocity $v_{\text {ej }}$ using $M_{\mathrm{ej}} \propto v_{\mathrm{ej}} \tau_{r}^{2}$ and $E_{\mathrm{kin}} \propto v_{\mathrm{ej}}^{3} \tau_{r}^{2}$ (e.g., Arnett 1982; Foley et al. 2009). Comparing PTF 09dav to a "typical" SN Ia with a kinetic energy of $10^{51} \mathrm{erg}$, a rise time of 17.4 days (Hayden et al. 2010), and a photospheric velocity of $11,000 \mathrm{~km} \mathrm{~s}^{-1}$ (Benetti et al. 2005), and assuming the opacities of both are the same, we estimate an ejecta mass of $0.36 M_{\odot}$ and kinetic energy of $8 \times 10^{49} \mathrm{erg}$. (Here, we have used an ejecta velocity of $6000 \mathrm{~km} \mathrm{~s}^{-1}$ for PTF 09dav estimated from the spectral fitting in Section 4.) These are low values; a typical ejecta mass for an $\mathrm{SN}$ Ia ranges from $\simeq 0.5 M_{\odot}$ for an $\mathrm{SN} 1991 \mathrm{bg}$-like event up to the Chandrasekhar mass $\left(1.4 M_{\odot}\right)$ for normal SNe Ia (e.g., Stritzinger et al. 2006; Mazzali et al. 2007). A similar low ejecta mass was derived for the calcium-rich SN Ib SN2005E (Perets et al. 2010), and even lower ejecta masses have been estimated for faint examples of SN2002cx-like events (Foley et al. 2009; McClelland et al. 2010) and for SN2002bj (Poznanski et al. 2010) and SN2010X (Kasliwal et al. 2010).

If the light curve is predominantly powered by the decay of ${ }^{56} \mathrm{Ni}$, the ${ }^{56} \mathrm{Ni}$ mass, $M_{\mathrm{Ni}}$, can be estimated from a knowledge of the rise time $\tau_{r}$ and $L_{\text {bol }}$ (Arnett 1982). At maximum light, $M_{\mathrm{Ni}}$ is given by $M_{\mathrm{Ni}}=L_{\mathrm{bol}} / \alpha \dot{S}\left(\tau_{r}\right)$ (Arnett's rule), where $\dot{S}$ is the radioactive luminosity per solar mass of ${ }^{56} \mathrm{Ni}$ from the decay to ${ }^{56} \mathrm{Co}$ to ${ }^{56} \mathrm{Fe}$ evaluated at maximum light and $\alpha$ is the ratio of bolometric to radioactive luminosities, near unity. We adopt $\dot{S}$ as defined in Howell et al. (2009; see also Branch 1992), which includes the effect of $\tau_{r}$, and take $\tau_{r}$ and its error from our light curve fits with a conservative additional two-day uncertainty in the rise-time error estimate to match the range found above. In practice, $\alpha$ is likely to deviate from unity. For normal SNe Ia, the light curve may peak at a luminosity that exceeds the instantaneous radioactive luminosity $(\alpha>1)$ due to a falling temperature and opacity (e.g., Branch 1992). On the other hand, for smaller ejecta masses some gamma rays may escape the ejecta without being thermalized (i.e., $\alpha<1$ ). Using the approach of Kasliwal et al. (2010), we estimate the optical depth of PTF 09dav at maximum light to be $\sim 70$ indicating an effective trapping of emitted $\gamma$-rays. For simplicity, we therefore take $\alpha=1$, giving $M_{\mathrm{Ni}}=0.019 \pm 0.003 M_{\odot}$. Under the same assumptions, SN 2008ha produced $0.0029 M_{\odot}$ of ${ }^{56} \mathrm{Ni}$, and SN 2007ax $0.038 \pm 0.008 M_{\odot}$-see Table 2 for estimated $M_{\mathrm{Ni}}$ for all the $1991 \mathrm{bg}$-like subluminous SNe. In these calculations, we estimated $\tau_{r}$ for each event by multiplying the stretch by the rise 


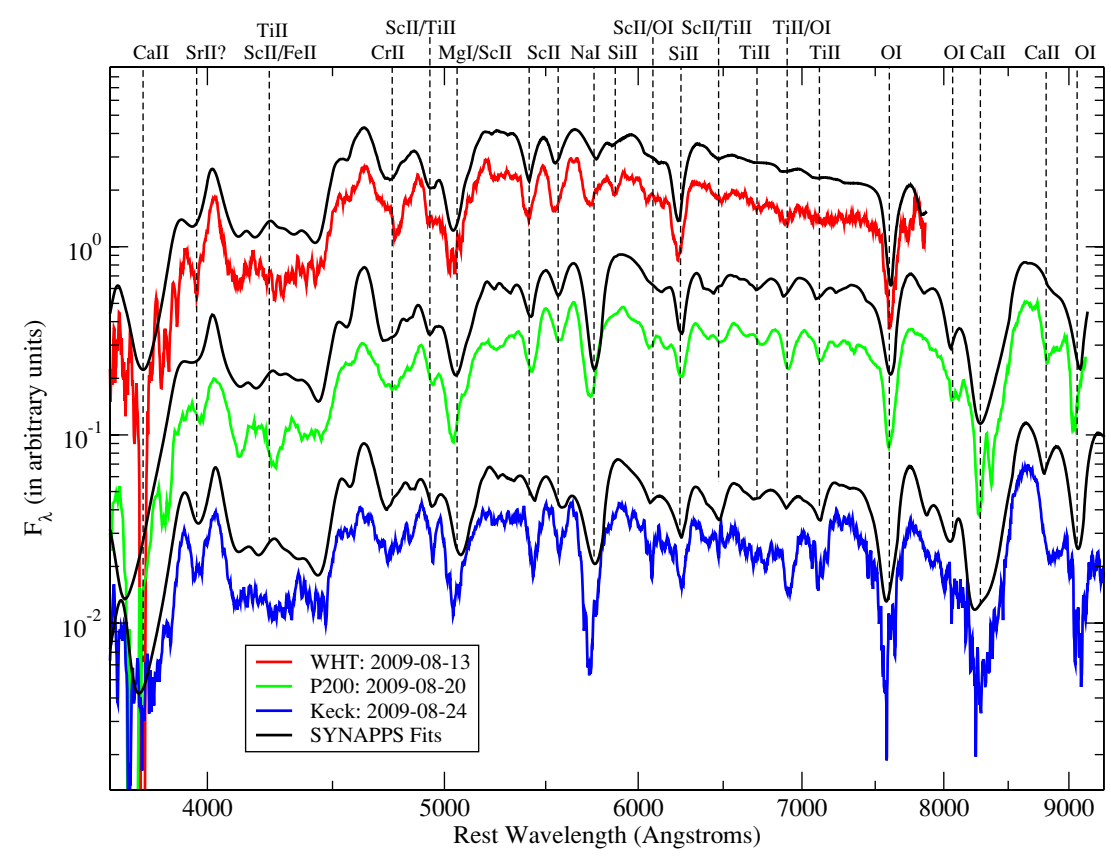

Figure 6. Three photospheric epoch spectra of PTF 09dav from WHT (+4.9 days), P200 (+11.2 days), and Keck (+16.0 days), along with their SYNAPPS fits. We have assumed a heliocentric redshift of 0.0371 . The major features in the spectrum are labeled at the minimum of their absorption. They include ionic species common in subluminous SNe Ia (O I, Ca II, Si II, Ti II) together with additional lines including Sc II and relatively strong Na I. We also tentatively identify Sr II and Cr II. Over the period in time covered by these spectra, the photospheric line velocities evolve from $\simeq 6100 \mathrm{~km} \mathrm{~s}^{-1}$ to $\simeq 5100 \mathrm{~km} \mathrm{~s}^{-1}$ to $\simeq 4600 \mathrm{~km} \mathrm{~s}^{-1}$.

(A color version of this figure is available in the online journal.)

time of the subluminous template (14 days). We emphasize that this calculation assumes the entire light curve to be powered by the decay of ${ }^{56} \mathrm{Ni}$.

In summary of this section, the $M_{B}, L_{\mathrm{bol}}, M_{\mathrm{Ni}}$, and ejecta mass $M_{\mathrm{ej}}$ of PTF 09dav are all unusually low. PTF 09dav is one of the faintest subluminous SNe Ia yet discovered, and, while not as faint as SN2008ha, it is the faintest of the SN1991bg-like sub-class.

\section{SPECTRAL ANALYSIS}

We now turn to the interpretation of the PTF 09dav spectra. The spectra of PTF 09dav show many curious features compared to other subluminous events (Figure 2). The comparison to SN2005bl (Taubenberger et al. 2008) is particularly instructive, as that spectrum was taken at an identical light curve phase (+5 days). Despite the resemblance to subluminous SNe Ia, with obvious Ti II, Si II, and O I absorption, other features in the spectra do not appear in other subluminous events (Figure 2). In particular, absorption features at $3960 \AA, 5400 \AA, 5540 \AA$, $5720 \AA, 6480 \AA, 6700 \AA$, and $6890 \AA$ do not, at first glance, immediately correspond to features in other subluminous SNe Ia. We attempt to identify these lines using an implementation of the SYNOW code and then discuss the results.

\subsection{Spectral Fitting}

When no detailed ab initio SN explosion model provides an immediate explanation for observations, parametrized spectrum synthesis provides the first step toward their construction. The idea is to use simplified radiative transfer calculations to directly fit SN spectra. A good fit constrains explosion models through interpretive spectral feature identification, with the main result being the detection or exclusion of specific chemical elements. The velocity distribution of detected species within the ejecta can also be constrained.
In our analysis we make use of SYNAPPS (Thomas et al. 2011). The physical assumptions SYNAPPS uses match those of the well-known SYNOW code (Fisher 2000), so findings are restricted to identification of features and not quantitative abundances. But where SYNOW is completely interactive, SYNAPPS is automated. This relieves the user from tedious, iterative adjustment of a large number of parameters (over 50 variables) to gain fit agreement, and assures more exhaustive searching of the parameter space. SYNAPPS can be thought of as the hybridization of a SYNOW-like calculation with a parallel optimization framework, where spectral fit quality serves as the objective function to optimize.

\subsection{Spectral Line Identifications}

We run SYNAPPS on our three photospheric spectra, and the resulting fits are shown in Figure 6. We identify the following lines common to subluminous SN Ia spectra: O I, Ca II, Si II, Ti II, Fe II, and Co II. The Ti II lines are particularly strong relative to other SN1991bg-like events. We also identify Sc II, Na I, and $\mathrm{Mg}$ I, as well as evidence for $\mathrm{Sr}$ II and possibly $\mathrm{Cr}$ II. There was no evidence for $\mathrm{S}$ II or $\mathrm{Mg}$ II-both degraded the quality of the fits, S II around $5500 \AA$, and $\mathrm{Mg}$ II in the red.

Though unusual, the presence of Sc II seems robust. As well as the two features at $5400 \AA$ and $5550 \AA$ (caused by $\lambda 5527$ and $\lambda 5658$ ), the $6490 \AA$ feature (caused by $\lambda 6604$ ), together with improved fits between 4000 and $5000 \AA$, provides additional confirmation. S II has strong lines in this region, but cannot be responsible for the two features at $5400 \AA$ and $5550 \AA$ (observer frame) - the ratio of the two line wavelengths does not match S II and the velocity would be inconsistent with the other elements (Figure 7). S II is common in 1991bg-like events (although the lines typically become weaker and disappear after maximum light), and its non-detection here is therefore surprising, but it does have a higher ionization energy than the other elements, which may point to lower temperatures. 

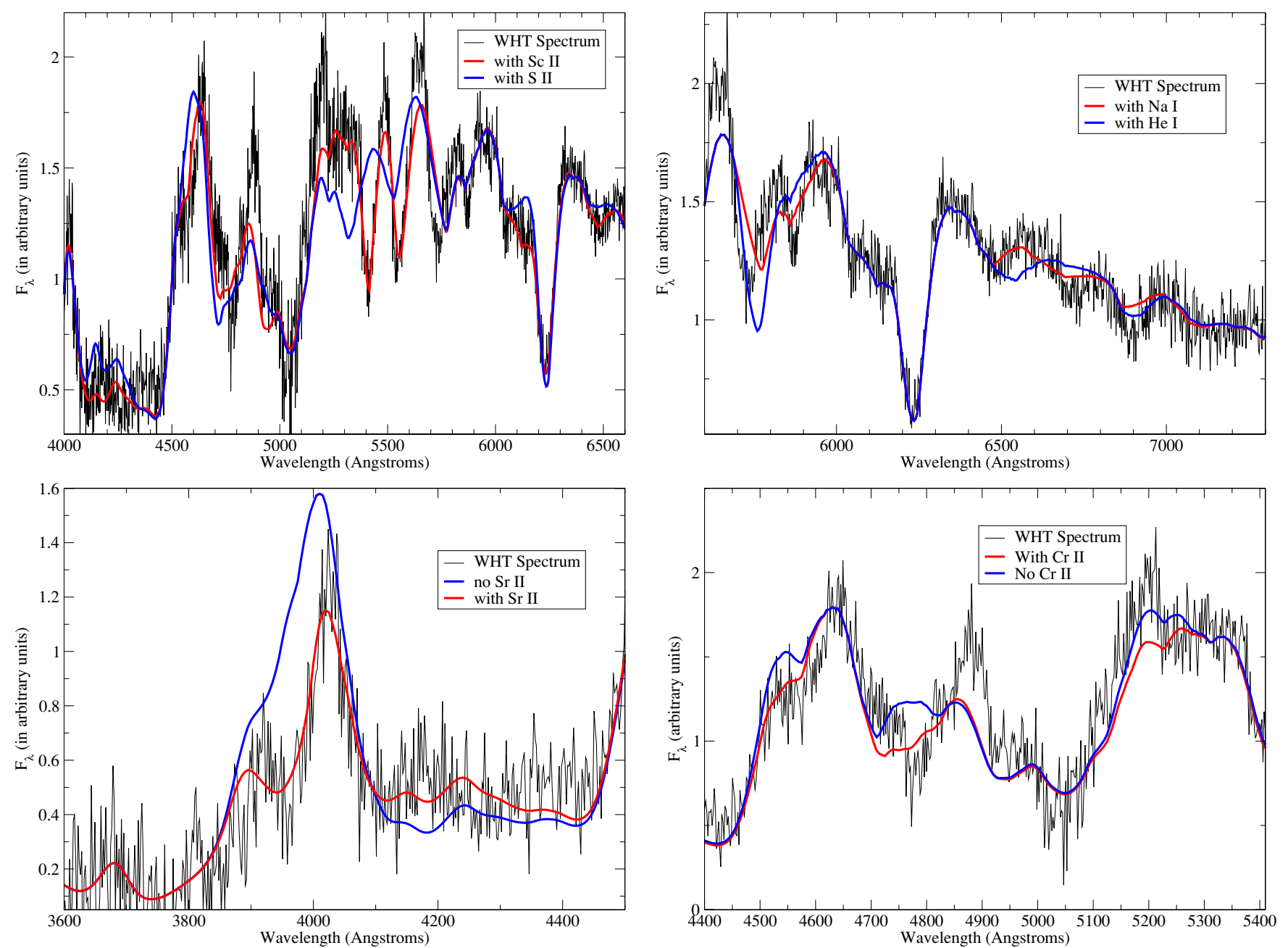

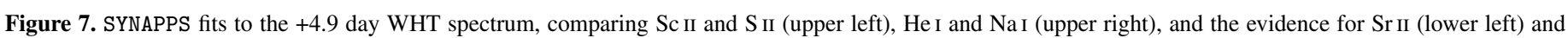

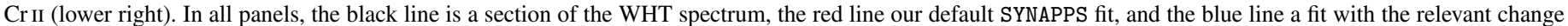

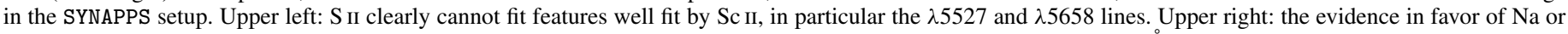

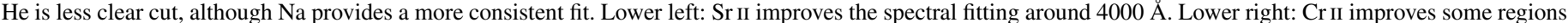
of the fit (especially around $4800 \AA$ ), but degrades the fit in other areas.

(A color version of this figure is available in the online journal.)

Although Sc II has been included in the SYNOW fits of faint SN2002cx-like SNe Ia (Foley et al. 2009; McClelland et al. 2010), it has never been robustly detected in SNe Ia. Sc II has been observed (together with the other $s$-process elements Ba II and Sr II) in SN1987A (Williams 1987; Mazzali et al. 1992) and also during the plateau phase of some lower-luminosity $\mathrm{SNe}$ IIP (Fassia et al. 1998; Pastorello et al. 2004). Mg I (with no indication of $\mathrm{Mg}$ II) is also surprising. As both Sc II and $\mathrm{Mg}$ I are only seen at low temperatures (Hatano et al. 1999), even lower than Ti II, this provides further evidence for the low temperatures of PTF 09dav.

NaI $\lambda 5892$ is also highly probable (Figure 7), and is typically seen in subluminous events after maximum light, although it is particularly strong in PTF 09dav-Figure 3. This feature could also be the He I $\lambda 5876$ line, and we cannot unambiguously confirm Na I over He I (Figure 7). Including $\mathrm{He}_{\mathrm{I}}$ in the fits does better match the $6900 \AA$ feature (produced by $\lambda 7065$ ), but it introduces other features in the SYNAPPS spectra which are not seen in the data, such as $6500 \AA$ (caused by $\lambda 6678$ ). However, SYNAPPS does not explicitly account for non-local thermodynamic equilibrium effects which are known to be important for modeling He I features (Lucy 1991)_LTE excitation in SYNAPPS can overestimate the strength of $\lambda 6678$ to $\lambda 5876$ and $\lambda 7065$ (Branch 2003). Thus, all we can say is that at least one of, and possibly both, $\mathrm{Na}$ I and $\mathrm{He}$ I are present in the spectra of PTF 09dav.

There is also reasonable evidence for the presence of $\mathrm{Sr}$ II (via absorption near $3960 \AA$ ), with weaker evidence for Cr II (primarily through absorption at $4770 \AA$ ), all shown in Figure 7 . Sr II is identified through a blended single line ( $\lambda 4078$ and $\lambda$ 4216), and has no other strong optical features. As with Sc II, Sr II is also only seen at low temperatures (Hatano et al. 1999). CrII is often weakly present in SN1991bg-like SNe (Mazzali et al. 1997; Taubenberger et al. 2008). Ba II, an $s$-process element often seen with Sr II, was not needed in the fits.

Given the apparent low temperatures, we also tested the inclusion of $\mathrm{S} \mathrm{I}_{\mathrm{I}}$ and $\mathrm{Si}$ I, but neither was required in the fits (their abundances came out to zero in SYNAPPS). Indeed, S I and Si I would add lines not seen in our spectra if their abundance were non-zero. We also experimented with $\mathrm{V}_{\text {II }}$ and $\mathrm{C}_{\text {II }}$ - there was no evidence for either in the fits, although they also did not degrade the fit results by adding extra lines not seen in the spectra. 


\subsection{Spectral Measurements}

We measure ejecta velocities from the blueshift of the absorption minima of the P-Cygni line profiles. This process can carry a number of uncertainties, particularly the blending of neighboring lines, so we use the SYNAPPS results which effectively average over several lines. The line velocities evolve from $\simeq 6100 \mathrm{~km} \mathrm{~s}^{-1}$ (+4.9 days) to $\simeq 5100 \mathrm{~km} \mathrm{~s}^{-1}$ (+11.2 days) to $\simeq 4600 \mathrm{~km} \mathrm{~s}^{-1}$ (+16.0 days), and the major lines all share a common velocity.

The strength of the $\mathrm{Na}$ I (or He I) line increases with time, and our tentative $\mathrm{Sr}$ II detection also becomes stronger. Ti II, already stronger than in most SN1991bg-like events, also increases in strength with phase. By contrast, the evidence for Fe II, as well as $\mathrm{Cr}$ II, has disappeared by +16 days; those lines are not needed in the later-epoch SYNAPPS fits.

We also estimate the Nugent et al. (1995) Si II ratio, $\mathcal{R}(\mathrm{Si})$, defined as the ratio of the depth of the Si II features at $\lambda 5972$ and $\lambda 6355$. We calculate this ratio as 0.35 , smaller than the values typical of subluminous $\mathrm{SNe}$ Ia of $\sim 0.65$ (Taubenberger et al. 2008), and naively indicating a higher temperature than in those events. However, the presence of strong emission from the $\mathrm{Na}$ I (or He I) line blueward of the $\lambda 5972$ feature will significantly affect this measurement, decreasing the depth of the $\lambda 5972$ feature and hence artificially decreasing the observed ratio. We also measure the (pseudo) equivalent widths of these two Si II features, which can be used to differentiate sub-types of SNe Ia using the W(6100)/W(5750) plane (Branch et al. 2006, 2009). Using the notation of Branch et al. (2006), W(6100) (the $\lambda 6355$ line) is $45 \AA$ and $\mathrm{W}(5750)$ (the $\lambda 5972$ line) is $10 \AA$. These measurements are both significantly lower than in SN1991bg-like events (typical values of $\simeq 100 \AA$ and $\simeq 45 \AA$, respectively), and therefore lie well outside the region in $\mathrm{W}(6100) / \mathrm{W}(5750)$ space containing other cool SNe with significant Ti II absorption (Branch et al. 2009).

\section{DISCUSSION}

PTF 09dav is an unusual supernova. Though superficially similar to a subluminous SN Ia, it is considerably fainter at $M_{B} \simeq-15.5$ (without being redder) and is one of the fastest $\mathrm{SNe}$ Ia observed. The peculiarity extends to its spectra, which have low ejecta velocities $\left(\sim 6000 \mathrm{~km} \mathrm{~s}^{-1}\right)$ and unusual spectral lines, including an unambiguous detection of Sc II-which we believe to be unique to PTF 09dav among SNe Ia. The origin of Sc is not clear. Though it is the decay product of ${ }^{44} \mathrm{Ti}$, the half-life is $\sim 60$ years, which makes that an unlikely source for the PTF 09dav maximum light spectra. ${ }^{45} \mathrm{Ti}$, with a halflife of $\sim 3$ hours, or higher and more unstable $\mathrm{Ti}$ isotopes, are more plausible if they are synthesized in large enough quantities. Some ${ }^{45} \mathrm{Ti}$ is predicted to be synthesized in the delayed detonation SN Ia explosion models of Maeda et al. (2010), but only with very small mass fractions. The low temperatures also probably contribute to the enhanced visibility of Sc. If real, the Sr II detection is also unusual and presumably originates from the $s$-process in the progenitor star.

PTF 09dav does share some similarities with SN2005E and the sub-class of calcium-rich Type $\mathrm{Ib} / \mathrm{c}$ SNe (Perets et al. 2010). SN2005E was faint $\left(M_{B}=-14.8\right)$, as are all calciumrich SNe, with a similar low ejecta mass to PTF 09dav (but higher ejecta velocities). SN2005E had a Ca-rich spectrum with no prominent intermediate-mass $\mathrm{C} / \mathrm{O}$-burning products (e.g., $\mathrm{Mg}, \mathrm{S}, \mathrm{Si}$ ), and although PTF 09dav is also $\mathrm{Ca} / \mathrm{Ti}$-rich, unlike SN2005E it does show the presence of Si and Mg lines.
Perhaps more interestingly, the environments are very similar. The environment of PTF 09dav is discrepant compared to other subluminous SNe Ia: PTF 09dav is either lying on the extreme outskirts of a spiral galaxy $(\sim 41 \mathrm{kpc}$ from the center, projected distance), in contrast to the massive E/S0 galaxies that form the bulk of the SN1991bg-like host population, or is located in a very faint, undetected dwarf host galaxy $(M>-12.8)$. Such a host galaxy would be one of the faintest on record; the SN1999aa-like SN Ia SN1999aw had a host absolute magnitude of $M_{V}=-12.4$ (Strolger et al. 2002), but this was a slowdeclining luminous event, in contrast to PTF 09dav. Similarly, SN2005E was also located some distance ( $\simeq 23 \mathrm{kpc}$ projected) from the center of its host galaxy, outside the plane of the disc, with no evidence for a faint dwarf host (or indeed any star formation activity) at the SN position.

The lack of a detection of a faint host galaxy at the position of PTF 09dav argues against a core-collapse origin and points to an older progenitor. The likelihood of finding a high-mass star far out in the galactic halo of a massive galaxy is discussed in detail by Perets et al. (2010), who conclude that a low-mass, old stellar progenitor is substantially more plausible at these large radii. A high-velocity and high-mass star ejected from the disk or center of the host of PTF 09dav is always conceivable, although an $8 M_{\odot}$ star would have to be ejected from the center at $\sim 1000 \mathrm{~km} \mathrm{~s}^{-1}$ to reach a distance of $41 \mathrm{kpc}$ in its $\sim 40 \mathrm{Myr}$ lifetime. While such stars have been observed in our galaxy (Brown et al. 2005; Edelmann et al. 2005; Heber et al. 2008), they are rare, and represent only a tiny fraction of massive stars in the Milky Way (Perets et al. 2010). They would also, by themselves, provide no explanation for the odd properties of PTF 09dav-the chances of PTF detecting an SN from such a star and it simultaneously having peculiar (unique) properties, seem low. Of course, the future detection of a faint host for PTF 09dav would certainly weight the argument in favor of a core-collapse origin-deeper imaging is underway (M. M. Kasliwal et al. 2011, in preparation).

At such a large galactic radius, the most likely progenitor type for PTF 09dav is an old (possibly metal-poor) white dwarf star, perhaps even located in a globular cluster (e.g., Pfahl et al. 2009). One simple physical explanation for PTF 09dav is that it is just a fainter example of a normal SN1991bg-like SN. The enhanced Ti II compared to brighter SN1991bg-like events, together with Sc II and Mg I and a lack of S II, point to lower temperatures consistent with the small inferred $M_{\mathrm{Ni}}$, and the light curve is well represented by a stretched version of SN1991bg. However, the SN also appears quite calcium-rich, particularly at later phases, with nebular [Ca II] $\lambda 7307$ beginning to emerge in the +16day spectrum, and an $i$-band excess consistent with strong [Ca II] emission at day +30 and beyond. This scenario also provides no immediate explanation for strong Sc II in the spectra (other than the cool temperatures that would at least allow any Sc II present to be seen), and, if the possible $\mathrm{He}_{\mathrm{I}}$ in the spectrum is real and not just $\mathrm{Na}$, then PTF 09dav is a very different event to other SN1991bg-like SNe.

Since calcium-rich spectra could arise from the detonation of He, Perets et al. (2010) propose that SN2005E-like SNe be explained by the detonation of helium on a CO core white dwarf (e.g., Nomoto 1982a, 1982b; Woosley et al. 1986; Woosley \& Weaver 1994; Livne \& Arnett 1995). These models are also worth exploring in the context of PTF 09dav. Various contemporary models of the observable outcomes of these sub-Chandrasekhar-mass explosions exist, with a variety of $\mathrm{CO}$ cores, ignition densities, and He shell masses modeled, 
and which consider both full star (CO core plus shell) and shell-only explosions (Bildsten et al. 2007; Shen et al. 2010; Sim et al. 2010; Fink et al. 2010; Waldman et al. 2011; Woosley \& Kasen 2011). The "full-star" explosions tend to be too luminous to explain the properties of subluminous $\mathrm{SNe}$, but the helium shell detonation models (Shen et al. 2010; Waldman et al. 2011) are more promising.

The light curves tend to be powered by the decay of ${ }^{56} \mathrm{Ni}$, ${ }^{48} \mathrm{Cr},{ }^{52} \mathrm{Fe}$ plus other radioactive nuclei and their decay products, but the key predicted observables of such scenarios can vary significantly according to the configuration of the progenitor system. Shen et al. (2010) consider CO cores of $>0.6 M_{\odot}$, and their models are typified by faint $\left(M_{R}=-15\right.$ to -18$)$, rapidly rising ( $<10$ days), and possibly multi-peaked light curves which redden quickly following maximum light due to the emissivity of Fe-group isotopes when they transition from the doubly to singly ionized state (e.g., Kasen 2006). The spectra are dominated by $\mathrm{Ca}$ II and Ti II features at high velocity $\left(\sim 10^{4} \mathrm{~km} \mathrm{~s}^{-1}\right)$, lacking intermediate mass elements (such as silicon). Lower CO core masses of $<0.6 M_{\odot}$ have been explored by Waldman et al. (2011), who show that at these low masses the abundance of ${ }^{56} \mathrm{Ni}$ drops sharply, with light curves powered predominantly by ${ }^{48} \mathrm{Cr}$, with the abundances of $\mathrm{Ca}$ and $\mathrm{Ti}$ both increasing together with the amount of unburnt helium. The presence of unburnt helium in the observed spectra of all He detonation models is unclear due to the LTE assumptions in the Shen et al. (2010) and Waldman et al. (2011) models, but some He might be expected.

The observations of PTF 09dav match several of these features. The spectra contain significant $\mathrm{Ca}$ II and Ti II. The light curve also peaks with a consistent luminosity, and becomes red in (e.g.,) $r-i$ at later phases, although this may also be plausibly explained by the emergence of the nebular [Ca II] doublet at $\lambda 7307$. The ejecta velocity and rise time are both on the edge of the model outcomes predicted by Shen et al. (2010), although more consistent with the lower CO core mass models of Waldman et al. (2011). However, there is no immediate explanation for the presence of Sc II (which is not present in any of the model spectra), and the spectra of PTF 09dav also contain both $\mathrm{Si}$ II and $\mathrm{O}$ I, which are predicted to have very low abundances in these helium detonation events. Thus, PTF 09dav is unlikely to be the result of a pure helium detonation, at least in the forms currently considered in the literature.

Another possibility for PTF 09dav is the deflagration of a helium shell layer on the surface of a $\mathrm{CO}$ white dwarf, which may occur for smaller He shell masses (Woosley \& Kasen 2011). These can have quite different properties to those of helium shell detonations, with the dim light curve $\left(M_{B} \sim-15\right)$ again powered mostly by ${ }^{48} \mathrm{Cr}$ (rather than ${ }^{56} \mathrm{Ni}$ ) leading to abundant $\mathrm{Ti}$ in the spectra. As the burning is incomplete, as with the detonation models some helium should also remain. The explosion energy per unit ejected mass is smaller than for the detonation models, giving slower light curves than for detonation models of the same brightness, with relatively low ejecta velocities $\sim 4000 \mathrm{~km} \mathrm{~s}^{-1}$. The presence of Sc in the spectra (from ${ }^{45} \mathrm{Ti}$ ) is a prediction in the helium deflagration models (Woosley \& Kasen 2011), although it is dependent on the neutron excess and hence metallicity. However, these deflagration models also predict quite blue optical colors ( $B-V \sim 0$ ) with peak luminosities fainter than PTF 09dav, and, as for the detonation models, there is a lack of $\mathrm{Si} / \mathrm{Mg}$ in the model spectra. In this regard, PTF 09dav seems to combine both $\mathrm{C}$-/O-burning and He-burning products-further detailed modeling and comparisons with observations will be required for a full understanding.

Whatever the physical explanation, PTF 09dav illustrates the discovery power of new high-cadence transient surveys such as PTF. Although the "faint and fast" observational parameter space remains relatively unexplored, these early new discoveries are able to test our understanding of cosmic explosions and motivate new directions in explosion modeling.

We acknowledge useful discussions with Brian Schmidt and Dan Kasen. We thank Richard Ellis for providing the P200/ DBSP spectrum. M.S. acknowledges support from the Royal Society. E.O.O. is supported by an Einstein fellowship and NASA grants. L.B. is supported by the National Science Foundation under grants PHY 05-51164 and AST 07-07633. S.B.C. acknowledges generous support from Gary and Cynthia Bengier and the Richard and Rhoda Goldman Foundation. D.P. is supported by an Einstein fellowship. The WHT is operated on the island of La Palma by the Isaac Newton Group in the Spanish Observatorio del Roque de los Muchachos of the Instituto de Astrofísica de Canarias. Observations obtained with the Samuel Oschin Telescope and the 60 inch Telescope at the Palomar Observatory as part of the Palomar Transient Factory project, a scientific collaboration between the California Institute of Technology, Columbia University, Las Cumbres Observatory, the Lawrence Berkeley National Laboratory, the National Energy Research Scientific Computing Center, the University of Oxford, and the Weizmann Institute of Science. Some of the data presented herein were obtained at the W. M. Keck Observatory, which is operated as a scientific partnership among the California Institute of Technology, the University of California, and the National Aeronautics and Space Administration. The Observatory was made possible by the generous financial support of the W. M. Keck Foundation. This research has made use of the NASA/IPAC Extragalactic Database (NED) which is operated by the Jet Propulsion Laboratory, California Institute of Technology, under contract with the National Aeronautics and Space Administration. The Weizmann Institute participation of in PTF is supported in part by grants from the Israeli Science Foundation to A.G. Weizmann-Caltech collaborative work on PTF is supported by a grant from the Binational Science Foundation (BSF) to A.G. and S.R.K. Collaborative work by A.G. and M.S. is supported by a grant from the Weizmann-UK "making connection" program. Collaborative work of A.G. and P.A.M. is supported by a Weizmann-Minerva grant. A.G. is supported by an EU/FP7 Marie Curie IRG fellowship and a research grant from the Peter and Patricia Gruber Awards.

Facilities: PO:1.2m, ING:Herschel, PO:1.5m, Hale, Keck:I, LCOGT

\section{REFERENCES}

Arcavi, I., et al. 2010, ApJ, 721, 777

Arnett, W. D. 1982, ApJ, 253, 785

Benetti, S., et al. 2005, ApJ, 623, 1011

Bessell, M. S. 1990, PASP, 102, 1181

Bildsten, L., Shen, K. J., Weinberg, N. N., \& Nelemans, G. 2007, ApJ, 662, L95 Branch, D. 1992, ApJ, 392, 35

Branch, D. 2003, in IAU Symp. 212, A Massive Star Odyssey: From Main Sequence to Supernova, ed. K. van der Hucht, A. Herrero, \& C. Esteban (San Francisco, CA: ASP), 346

Branch, D., Baron, E., Thomas, R. C., Kasen, D., Li, W., \& Filippenko, A. V. 2004, PASP, 116, 903

Branch, D., Dang, L. C., \& Baron, E. 2009, PASP, 121, 238

Branch, D., Fisher, A., \& Nugent, P. 1993, AJ, 106, 2383

Branch, D., et al. 2006, PASP, 118, 560 
Brown, W. R., Geller, M. J., Kenyon, S. J., \& Kurtz, M. J. 2005, ApJ, 622, 33 Cardelli, J. A., Clayton, G. C., \& Mathis, J. S. 1989, ApJ, 345, 245

Cenko, S. B., et al. 2006, PASP, 118, 1396

Conley, A., et al. 2008, ApJ, 681, 482

Contardo, G., Leibundgut, B., \& Vacca, W. D. 2000, A\&A, 359, 876

Edelmann, H., Napiwotzki, R., Heber, U., Christlieb, N., \& Reimers, D. 2005, ApJ, 634, L181

Ellis, R. S., et al. 2008, ApJ, 674, 51

Fassia, A., Meikle, W. P. S., Geballe, T. R., Walton, N. A., Pollacco, D. L., Rutten, R. G. M., \& Tinney, C. 1998, MNRAS, 299, 150

Filippenko, A. V., et al. 1992, AJ, 104, 1543

Fink, M., Röpke, F. K., Hillebrandt, W., Seitenzahl, I. R., Sim, S. A., \& Kromer, M. 2010, A\&A, 514, A53

Fisher, A. K. 2000, PhD thesis, Univ. Oklahoma

Foley, R. J., Brown, P. J., Rest, A., Challis, P. J., Kirshner, R. P., \& Wood-Vasey, W. M. 2010, ApJ, 708, L61

Foley, R. J., et al. 2009, AJ, 138, 376

Garnavich, P. M., et al. 2004, ApJ, 613, 1120

Gonzalez-Gaitan, S., et al. 2011, ApJ, 727, 107

Guy, J., et al. 2007, A\&A, 466, 11

Hatano, K., Branch, D., Fisher, A., Millard, J., \& Baron, E. 1999, ApJS, 121, 233

Hayden, B. T., et al. 2010, ApJ, 712, 350

Heber, U., Edelmann, H., Napiwotzki, R., Altmann, M., \& Scholz, R. 2008, A\&A, 483, L21

Hicken, M., et al. 2009, ApJ, 700, 331

Howell, D. A. 2001, ApJ, 554, L193

Howell, D. A., et al. 2005, ApJ, 634, 1190

Howell, D. A., et al. 2009, ApJ, 691, 661

Hsiao, E. Y., Conley, A., Howell, D. A., Sullivan, M., Pritchet, C. J., Carlberg, R. G., Nugent, P. E., \& Phillips, M. M. 2007, ApJ, 663, 1187

Kasen, D. 2006, ApJ, 649, 939

Kasliwal, M. M., et al. 2008, ApJ, 683, L29

Kasliwal, M. M., et al. 2010, ApJ, 723, L98

Law, N. M., et al. 2009, PASP, 121, 1395

Leibundgut, B., et al. 1993, AJ, 105, 301

Li, W., et al. 2003, PASP, 115, 453

Livne, E., \& Arnett, D. 1995, ApJ, 452, 62

Lucy, L. B. 1991, ApJ, 383, 308

Maeda, K., Röpke, F. K., Fink, M., Hillebrandt, W., Travaglio, C., \& Thielemann, F. 2010, ApJ, 712, 624

Mazzali, P. A., Chugai, N., Turatto, M., Lucy, L. B., Danziger, I. J., Cappellaro, E., della Valle, M., \& Benetti, S. 1997, MNRAS, 284, 151
Mazzali, P. A., Lucy, L. B., \& Butler, K. 1992, A\&A, 258, 399

Mazzali, P. A., Röpke, F. K., Benetti, S., \& Hillebrandt, W. 2007, Science, 315 , 825

McClelland, C. M., et al. 2010, ApJ, 720, 704

Moriya, T., Tominaga, N., Tanaka, M., Nomoto, K., Sauer, D. N., Mazzali, P. A., Maeda, K., \& Suzuki, T. 2010, ApJ, 719, 1445

Neill, J. D., et al. 2009, ApJ, 707, 1449

Nomoto, K. 1982a, ApJ, 257, 780

Nomoto, K. 1982b, ApJ, 253, 798

Nugent, P., Kim, A., \& Perlmutter, S. 2002, PASP, 114, 803

Nugent, P., Phillips, M., Baron, E., Branch, D., \& Hauschildt, P. 1995, ApJ, 455, L147

Nugent, P. E., Transient Factory, P., \& Project, D. 2009, BAAS, 41, 419

Oke, J. B., \& Gunn, J. E. 1982, PASP, 94, 586

Oke, J. B., \& Gunn, J. E. 1983, ApJ, 266, 713

Oke, J. B., et al. 1995, PASP, 107, 375

Pastorello, A., et al. 2004, MNRAS, 347, 74

Perets, H. B., Badenes, C., Arcavi, I., Simon, J. D., \& Gal-Yam, A. 2011, ApJ, 730, 89

Perets, H. B., et al. 2010, Nature, 465, 322

Pfahl, E., Scannapieco, E., \& Bildsten, L. 2009, ApJ, 695, L111

Phillips, M. M. 1993, ApJ, 413, L105

Phillips, M. M., et al. 2007, PASP, 119, 360

Poznanski, D., et al. 2010, Science, 327, 58

Rau, A., et al. 2009, PASP, 121, 1334

Riess, A. G., Filippenko, A. V., Li, W., \& Schmidt, B. P. 1999, AJ, 118, 2668

Schlegel, D. J., Finkbeiner, D. P., \& Davis, M. 1998, ApJ, 500, 525

Shen, K. J., Kasen, D., Weinberg, N. N., Bildsten, L., \& Scannapieco, E. 2010, ApJ, 715, 767

Sim, S. A., Röpke, F. K., Hillebrandt, W., Kromer, M., Pakmor, R., Fink, M., Ruiter, A. J., \& Seitenzahl, I. R. 2010, ApJ, 714, L52

Stritzinger, M., Leibundgut, B., Walch, S., \& Contardo, G. 2006, A\&A, 450, 241

Strolger, L.-G., et al. 2002, AJ, 124, 2905

Taubenberger, S., et al. 2008, MNRAS, 385, 75

Thomas, R. C., Nugent, P. E., \& Meza, J. C. 2011, PASP, 123, 237

Valenti, S., et al. 2009, Nature, 459, 674

Waldman, R., Sauer, D., Livne, E., Perets, H., Glasner, A., Mazzali, P., Truran, J. W., \& Gal-Yam, A. 2011, arXiv:1009.3829

Williams, R. E. 1987, ApJ, 320, L117

Woosley, S. E., \& Kasen, D. 2011, arXiv:1010.5292

Woosley, S. E., Taam, R. E., \& Weaver, T. A. 1986, ApJ, 301, 601

Woosley, S. E., \& Weaver, T. A. 1994, ApJ, 423, 371 